\title{
On the evaluation of elastic critical moments in doubly and singly symmetric I-section cantilevers
}

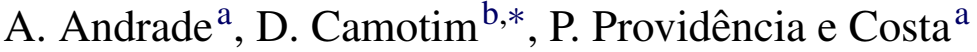 \\ ${ }^{a}$ Department of Civil Engineering, University of Coimbra, Pólo II, Pinhal de Marrocos, 3030-290 Coimbra, Portugal \\ ${ }^{\mathrm{b}}$ Department of Civil Engineering and Architecture, ICIST/IST, Technical University of Lisbon, Av. Rovisco Pais, 1049-001 Lisboa, Portugal
}

Received 15 June 2006; accepted 31 August 2006

\begin{abstract}
The so-called 3-factor formula is one of the most commonly employed general formulae to estimate the elastic critical moment of steel beams prone to lateral-torsional buckling. This work extends its domain of application to I-section cantilevers (i) with equal or unequal flanges, (ii) fully built-in or free to warp at the support and (iii) acted on by uniformly distributed or concentrated tip loads (applied either at the shear centre or at one of the flanges). The paper includes (i) a discussion of the theoretical basis of elastic lateral-torsional buckling, (ii) the description of the main steps involved in posing the buckling problem in a non-dimensional form over a fixed reference domain, features that are particularly convenient for the purpose of this work, (iii) the numerical results of a parametric study, obtained by the Rayleigh-Ritz method, and (iv) their use for the development of approximate analytical expressions for the $C_{1}, C_{2}$ and $C_{3}$ factors appearing in the aforementioned formula.
\end{abstract}

(c) 2006 Elsevier Ltd. All rights reserved.

Keywords: Lateral-torsional buckling; Elastic critical moments; I-section cantilevers; Dimensional analysis; Rayleigh-Ritz method; Eurocode 3

\section{Introduction}

According to virtually all the design codes currently employed in the steel construction industry (e.g., [1-4]), the load carrying capacity of laterally unsupported beams bent in their stiffer principal plane is estimated on the basis of their (i) cross-sectional (direct stresses) and (ii) elastic lateraltorsional buckling (LTB) resistances-therefore, designers must have easy access to reliable methods for computing the elastic critical moment or load of a given beam. Of course, this calculation can be carried out by means of a number of numerical techniques, which basically involve the approximation of either (i) the individual terms in the governing differential equations (e.g., finite difference methods) or (ii) the function spaces associated with an underlying variational principle (e.g., finite element methods). However, until userfriendly software packages implementing such numerical procedures become widely available to practitioners, simplified methods such as design charts and approximate formulae will retain their popularity and continue to be extensively used.

\footnotetext{
* Corresponding author. Tel.: +351 21 8418403; fax: +351 218497650

E-mail address: dcamotim@ civil.ist.utl.pt (D. Camotim).
}

One of the most commonly employed general formulae to estimate elastic critical moments $\left(M_{c r}\right)$ is the so-called 3-factor formula, which was included in the ENV version of Eurocode 3 [5] - the recently completed EN version of this design standard [1] provides no information concerning the determination of $M_{c r}$. In theory, this formula should be applicable to uniform beams (or beam segments) subjected to major axis bending, exhibiting doubly or singly symmetric cross-sections (with the proviso that the cross-sections are symmetric with respect to the minor central axis) and arbitrary support and loading conditions. But some situations are currently not covered, most notably the case of cantilevers, which are free to twist and deflect laterally at one endinformation about the factors that must be introduced in the formula is rather scarce and/or incomplete. In a 1960 review article, Clark and Hill [6] presented lower and upper bounds for the $C_{1}$ factor applicable to cantilevers restrained from warping at the fixed end and acted on by a uniform load or by a point load applied at the tip-in the latter case, a $C_{2}$ factor was also proposed. Moreover, $C_{1}$ and $C_{2}$ factors for these two types of loading were also published by Galéa [7] and Baláz and Koleková [8], but the warping restraint condition at the support was not specified. However, the authors did 
not find in the literature any proposal for the $C_{3}$ factor, a fact that automatically rules out the possibility of dealing with singly symmetric cantilevers. At this point, a word of caution is required: there are slight differences in the formulae developed by the above authors-for instance, none of the formulae proposed by Clark and Hill or Galéa contain an effective length factor $k_{w}$, unlike the formula included in [5] and also considered by Baláz and Koleková.

Over the years, a fair amount of research work has been devoted to develop approximate formulae to assess the buckling behaviour of I-beams. In the following paragraphs, a brief state-of-the-art survey on this subject is presented, focusing specifically on cantilevers.

The elastic LTB of equal-flanged I-section cantilevers built-in at the support and under shear centre loading was investigated by Timoshenko [9] (point load at the tip) and by Poley [10] (uniform load). In the 70s, Nethercot [11] extended these early works by considering the effects of (i) top flange loading, (ii) the type of restraint condition at the tip and (iii) the continuity between overhanging and internal segments-in addition, this author proposed a set of effective length factors to estimate $M_{c r}$. On the other hand, Trahair [12] developed approximate formulae to estimate the elastic buckling capacity of built-in cantilevers and of overhanging segments free to warp at the support, without any interaction with the internal segments-he also proposed a simplified method to account for the interaction between adjacent segments and studied the effect of the presence of an elastic torsional restraint at the support. However, the above studies were restricted to doubly symmetric I-section members-note that the elastic critical moment of doubly symmetric cantilevers free to warp at the support can also be estimated by a single factor formula included in a recently published design guide [13]. By applying curve fitting techniques to a collection of finite element results, Doswell [14] proposed an improvement of the approximate $M_{c r}$ formula included in the current AISC specification [3]-this proposal takes explicitly into account, through individual coefficients, the effects of (i) the bending moment diagram (two loading case are analysed: tip load and uniform load), (ii) the level of load application (only the cases of shear centre and top flange loading are considered) and (iii) the effect of top flange bracing (either continuous bracing or a discrete bracing at the free end). Once more, only equal-flanged I-sections were dealt with and the cantilevers are always assumed to be fully built-in at the support.

The elastic LTB of singly symmetric I-section cantilevers fully built-in at the support and acted on by a tip load or a uniform load was studied by Anderson and Trahair [15], Roberts and Burt [16] and Wang and Kitipornchai [17], who provided tables, charts and/or approximate expressions to calculate elastic critical moments/loads. Moreover, the effect of intermediate restraints was investigated by Wang et al. [18].

This paper attempts to fill-in two of the insufficiencies identified in the above studies, by (i) examining the influence of the warping restraint condition on the elastic LTB of singly symmetric cantilevers and (ii) extending the domain of application of the 3 -factor formula to I-section cantilevers ( $\mathrm{ii}_{1}$ )



(a)

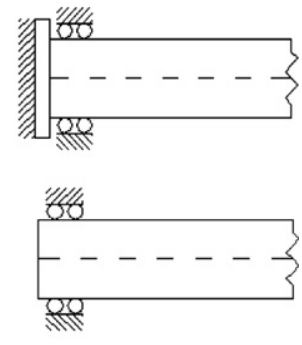

(b)
Fig. 1. (a) Undeformed singly symmetric I-section cantilever and external loads. (b) Schematic representation of the warping conditions at the fixed end.

with equal or unequal flanges, ( $\mathrm{ii}_{2}$ ) fully built-in or free to warp at the support and $\left(\mathrm{ii}_{3}\right)$ acted by uniform or tip loads (applied at one of the flanges or at the shear centre), thus making available to practitioners a reliable and useful design tool. The paper includes a discussion of the theoretical basis of elastic LTB, as well as the description of the main steps involved in posing the buckling problem in a non-dimensional form over a fixed reference domain - a particularly adequate setting for the systematic development of approximate buckling formulae. This approach, which has its roots in dimensional analysis, differs significantly from the one proposed by Attard [19], who has non-dimensionalised a general expression for $M_{c r}$ written in terms of an assumed mode shape. Finally, note that preliminary and partial versions of this work were reported in [20-22].

\section{Theoretical background}

\subsection{Problem statement}

We consider a prismatic cantilever with a doubly or singly symmetric I-section (i.e. equal or unequal flanges) and length $l$, as the one shown in Fig. 1(a). It is convenient to introduce a fixed rectangular right-handed Cartesian reference system such that, in the undeformed configuration, (i) the longitudinal centroidal axis of the beam coincides with the $x$-axis, (ii) $y$ and $z$ are the cross-section major and minor central axes and (iii) the top flange corresponds to negative $z$ values (see Fig. 1(a)) thus, $y=0$ is a symmetry plane for the undeformed member. The end cross-sections are initially contained in the planes $x=$ 0 and $x=l$ and the locus of shear centres is the straight-line segment defined by $\left\{(x, y, z) \in \mathrm{R}^{3}: x \in[0, l], y=0, z=z_{S}\right\}$. With no loss of generality, we assume that $x=0$ corresponds to the fixed end section, where the lateral deflections (along $y$ ), minor axis rotations (about $z$ ) and torsional rotations (about the shear centre axis) are prevented and warping is either fully restrained or completely free- - these two warping conditions at the fixed end are schematically represented in Fig. 1(b) and will be subsequently identified by the acronyms $\mathrm{NW}$ and $\mathrm{FW}$, which stand for No Warping and Free Warping, respectively.

The cantilever is made of a Saint-Venant/Kirchhoff material [23], with Young modulus $E$ and shear modulus $G$. It is deemed to be subjected to the conservative transverse loads depicted in Fig. 1(a) - a uniform load $\boldsymbol{q}=q \boldsymbol{k}$ and a 
tip load $\boldsymbol{Q}=Q \boldsymbol{k}$, where $\boldsymbol{k}$ is the unit vector directed along the $z$-axis - , both with the same direction and initially acting on the plane $y=0$. Their magnitudes $q, Q$ are assumed to be proportional to a single factor $\lambda$ (therefore, we may write $q=q_{0} \lambda$ and $Q=Q_{0} \lambda$, where $q_{0}, Q_{0}$ are non-negative reference magnitudes defining the loading profile) and their conservative character is ensured by the fact that they follow the beam deformation, always remaining parallel to the $z$-axis. For convenience, we also assume throughout this section that $q_{0}$ and $Q_{0}$ are strictly positive.

It is well-known that such a cantilever is prone to lateraltorsional buckling, a bifurcation-type of instability (from the Latin word bifurcus- 'with two branches') where (i) the fundamental path corresponds to equilibrium configurations (parameterised by $\lambda$ ) that are symmetric with respect to the plane $y=0$ (i.e. the cantilever is subjected solely to major axis bending) and (ii) the buckled states are associated with non-symmetric configurations - the cantilever deflects laterally, along $y$, and twists. This phenomenon is an obvious instance of symmetry breaking. However, not all symmetry is lost: given any possible buckled state, its reflection upon the plane $y=0$ is also a possible buckled state-i.e. instability breaks the symmetry of the equilibrium configuration, but not the symmetry of the solution set.

In this section, we aim at formulating the problem of identifying the bifurcation points along the cantilever's fundamental path in a way that is well suited for the systematic development of approximate buckling formulae. In order to linearise the problem, we neglect the pre-buckling flexural deflections, thus assuming that the cantilever remains straight up until the onset of buckling - in design, it is prudent to disregard the beneficial effect of pre-buckling deflections because (i) it may be significantly reduced, or even fully removed, by any pre-cambering of the member and (ii) it depends heavily on the actual conditions of lateral restraint [24]. Moreover, the analysis is carried out under the assumption of small strains.

\subsection{Mathematical modelling}

Using Vlassov's classical thin-walled beam theory hypotheses [25] - the cross-sections do not deform in their own plane and the shear strains on the mid-surface are negligible - , it is possible to show that the second variation, from a given fundamental state, of the cantilever total potential energy $\Pi$ is defined by (e.g., [26])

$$
\begin{aligned}
\delta^{2} \Pi= & \frac{E I_{z}}{2} \int_{0}^{l} v_{, x x}^{2} \mathrm{~d} x+\frac{G I_{t}}{2} \int_{0}^{l} \phi_{, x}^{2} \mathrm{~d} x+\frac{E I_{w}}{2} \int_{0}^{l} \phi_{, x x}^{2} \mathrm{~d} x \\
& +\frac{1}{2} \int_{0}^{l} M_{y}^{f}\left(2 v_{, x x} \phi+\beta_{y} \phi_{, x}^{2}\right) \mathrm{d} x+\frac{1}{2}\left(z_{q}-z_{S}\right) q \\
& \times \int_{0}^{l} \phi^{2} \mathrm{~d} x+\frac{1}{2}\left(z_{Q}-z_{S}\right) Q \phi(l)^{2},
\end{aligned}
$$

where (i) $v(x)$ and $\phi(x)$ are variations of $V(x, \lambda)$ (shear centre displacement along $y$ ) and $\Phi(x, \lambda)$ (rotation about the undeformed shear centre axis), which are independent from $\lambda$ (recall that $V$ and $\Phi$ are both identically zero in the fundamental state), (ii) $I_{z}, I_{t}$ and $I_{w}$ are standard geometrical properties of the undeformed cross-section, (iii) $\beta_{y}$ is a cross-sectional asymmetry property, defined by

$\beta_{y}=\frac{1}{I_{y}} \int_{A} z\left(y^{2}+z^{2}\right) \mathrm{d} A-2 z_{S}$,

(iv) $M_{y}^{f}$ denotes the bending moment distribution in the fundamental state, given by

$$
\begin{aligned}
M_{y}^{f}(x, \lambda) & =-\left(\frac{1}{2} q_{0}(l-x)^{2}+Q_{0}(l-x)\right) \lambda \\
& =M_{y 0}^{f}(x) \lambda,
\end{aligned}
$$

where $M_{y 0}^{f}$ is a function of $x$ alone and denotes the bending moment diagram caused by the reference loads $q_{0} \boldsymbol{k}$ and $Q_{0} \boldsymbol{k}$, and (v) $z_{q}$ and $z_{Q}$ identify the point of load applicationsee Fig. 1(a). In the sequel, we exclude the limiting cases of $\mathrm{T}$, inverted $\mathrm{T}$ and narrow rectangular cross-sections, so as to ensure that $I_{w} \neq 0$.

In order to completely define the functional $\delta^{2} \Pi$, it is necessary to specify the class of admissible functions $v$ and $\phi$. There are two properties that must be characterised: (i) the smoothness required of these functions and (ii) the boundary conditions they must satisfy. The integrals in (1) only make sense if $v$ and $\phi$ are square-integrable in $(0, l)$ and possess square-integrable first and second derivatives. In addition, the admissible functions $v$ and $\phi$ must satisfy the homogeneous form of the essential (Dirichlet) boundary conditions, i.e.

$v(0)=0 \quad v_{, x}(0)=0 \quad \phi(0)=0 \quad \phi_{, x}(0)=0$,

the last one applying only if warping is prevented at the fixed end section (NW). The real-valued functions fulfilling the above requirements are termed kinematically admissible.

According to Trefftz's criterion [27,28] - for a critical appraisal of this criterion, together with a brief historical account and references to its application, the interested reader is referred to [29] -, the variational form of the buckling problem can be stated as follows:

Problem A (Variational Form). Find real scalars $\lambda$ and kinematically admissible functions $v, \phi \neq 0$ rendering $\delta^{2} \Pi$ stationary, i.e. satisfying the variational condition

$\delta\left(\delta^{2} \Pi\right)=0$.

Applying standard Calculus of Variations techniques [30], we obtain the classical or strong form of Problem A, which may be phrased as follows:

Problem A (Strong Form). Find $\lambda \in \mathrm{R}$ and real-valued functions $v, \phi \in C^{4}([0, l])$, with $v, \phi \neq 0$, satisfying the differential equations

$$
\begin{aligned}
& E I_{z} v_{, x x x x}+\left(M_{y 0}^{f} \phi\right)_{, x x} \lambda=0 \\
& \left(M_{y 0}^{f} v_{, x x}+q_{0}\left(z_{q}-z S\right) \phi-\beta_{y}\left(M_{y 0}^{f} \phi_{, x}\right)_{, x}\right) \lambda-G I_{t} \phi_{, x x} \\
& \quad+E I_{w} \phi_{, x x x x}=0
\end{aligned}
$$


in $(0, l)\left(\right.$ Euler-Lagrange equations of $\left.\delta^{2} \Pi\right)$ and the boundary conditions

$$
\begin{aligned}
& v(0)=0 \quad Q_{0} \phi(l) \lambda+E I_{z} v_{, x x x}(l)=0 \\
& v_{, x}(0)=0 \quad E I_{z} v_{, x x}(l)=0 \\
& \phi(0)=0 \quad\left(z_{Q}-z_{S}\right) Q_{0} \phi(l) \lambda+G I_{t} \phi_{, x}(l) \\
& \quad-E I_{w} \phi_{, x x x}(l)=0 \\
& \phi_{, x}(0)=0 \quad(\mathrm{NW}) \quad \text { or } \quad E I_{w} \phi_{, x x}(0)=0(\mathrm{FW}) \\
& E I_{w} \phi_{, x x}(l)=0 .
\end{aligned}
$$

Due to the regularity of the data - constant mechanical and geometrical properties and $M_{y 0}^{f} \in C^{\infty}([0, l])$-, the strong and variational forms of the problem are equivalent.

From a mathematical viewpoint, Problem A is an eigenvalue problem - the elastic bifurcation load factors and the corresponding buckling modes are its eigenvalues and eigenfunctions. Given the self-adjoint character of this problem, all eigenvalues are real and the eigenfunctions may be taken to be real [31,32] - this is the mathematical justification for having formulated Problem A in terms of real scalars and real function spaces, in line with its physical nature. The lowest positive eigenvalue is termed elastic critical load factor and denoted by $\lambda_{c r}$-accordingly, the corresponding buckling mode $\left(v_{c r}, \phi_{c r}\right)$ is labelled critical as well. We also speak of the elastic critical moment, defined as

$$
\begin{aligned}
M_{c r} & =\left|M_{y}^{f}\left(x, \lambda_{c r}\right)\right|_{\max }=\left(1 / 2 q_{0} l^{2}+Q_{0} l\right) \lambda_{c r} \\
& =-M_{y 0}^{f}(0) \lambda_{c r} .
\end{aligned}
$$

Integrating twice the field equation (6), together with the natural (Neumann) boundary conditions $\left(8_{2}\right)-\left(9_{2}\right)$, we are led to the conclusion that $v_{, x x}$ and $\phi$ are related by the equation

$v_{, x x}=-\frac{M_{y 0}^{f}}{E I_{z}} \phi \lambda$

This equation can be viewed as a holonomic kinematical constraint [33], which can be used to eliminate $v$ and write the buckling problem in terms of a single unknown field $\phi$. Indeed, the incorporation of (12) into (7) yields

$$
\begin{aligned}
- & \frac{M_{y 0}^{f 2}}{E I_{z}} \phi \lambda^{2}+\left(q_{0}\left(z_{q}-z_{S}\right) \phi-\beta_{y}\left(M_{y 0}^{f} \phi_{, x}\right)_{, x}\right) \lambda \\
& -G I_{t} \phi_{, x x}+E I_{w} \phi_{, x x x x}=0
\end{aligned}
$$

and Problem A may then be replaced by the following one, which takes on the form of a quadratic eigenvalue problem:

Problem B (Strong Form). Find $\lambda \in \mathrm{R}$ and $\phi \in C^{4}([0, l])$, with $\phi \neq 0$, satisfying the field equation (13) and the boundary conditions (10) and (11).

It is obvious that a variational characterisation can be given for Problem B: it suffices to introduce (12) into (1) and to consider again Eq. (5). If required, $v$ can be obtained through the mere integration of Eq. (12) subjected to the initial conditions $\left(8_{1}\right)-\left(9_{1}\right)$.

\subsection{Equivalent scaled problems}

By an appropriate change of variable and subsequent scaling of the functions $v$ and $\phi$, Problems A and B may be transformed into equivalent ones, posed in a fixed reference domain (i.e. independent of the cantilever length $l$ ) and written in a nondimensional form - this is most convenient because it enables a general and systematic representation of the cantilever LTB behaviour.

Since the interval $[0,1]$ is taken as the fixed reference domain, the associated change of variable is defined by the stretch $\xi:[0, l] \rightarrow[0,1], \xi=x / l$. Moreover, we introduce the scaled non-dimensional functions $\bar{v}:[0,1] \rightarrow \mathrm{R}, \bar{v}(\xi)=$ $\sqrt{E I_{z} /\left(G I_{t}\right)} v(\xi l) / l$ and $\bar{\phi}:[0,1] \rightarrow \mathrm{R}, \bar{\phi}(\xi)=\phi(\xi l)$. Then, the strong form of the scaled versions of Problems A and B may be stated as follows:

Problem C (Strong Form). Find $\lambda \in \mathrm{R}$ and $\bar{v}, \bar{\phi} \in C^{4}([0,1])$, with $\bar{v}, \bar{\phi} \neq 0$, satisfying the differential equations

$$
\begin{aligned}
& \bar{v}_{, \xi \xi \xi \xi}+\left(\mu_{0}^{f} \bar{\phi}\right)_{, \xi \xi} \lambda=0 \\
& \left(\mu_{0}^{f} \bar{v}_{, \xi \xi}+2 \varepsilon_{q} \gamma_{q} \bar{\phi}-\delta_{y}\left(\mu_{0}^{f} \bar{\phi}_{, \xi}\right)_{, \xi}\right) \lambda-\bar{\phi}_{, \xi \xi} \\
& \quad+\frac{K^{2}}{\pi^{2}} \bar{\phi}_{, \xi \xi \xi \xi}=0
\end{aligned}
$$

in $(0,1)$ and the boundary conditions

$$
\begin{aligned}
& \bar{v}(0)=0 \quad \gamma_{Q} \bar{\phi}(1) \lambda+\bar{v}_{, \xi \xi \xi}(1)=0 \\
& \bar{v}_{, \xi}(0)=0 \quad \bar{v}_{, \xi \xi}(1)=0 \\
& \bar{\phi}(0)=0 \quad \varepsilon_{Q} \gamma_{Q} \bar{\phi}(1) \lambda+\bar{\phi}_{, \xi}(1)-\frac{K^{2}}{\pi^{2}} \bar{\phi}_{, \xi \xi \xi}(1)=0 \\
& \bar{\phi}_{, \xi}(0)=0(\mathrm{NW}) \quad \text { or } \quad \bar{\phi}_{, \xi \xi}(0)=0(\mathrm{FW}) \quad \bar{\phi}_{, \xi \xi}(1)=0,
\end{aligned}
$$

where

$$
\begin{aligned}
\mu_{0}^{f}(\xi) & =\frac{l}{\sqrt{E I_{z} G I_{t}}} M_{y 0}^{f}(\xi l) \\
& =-(1-\xi)\left((1-\xi) \gamma_{q}+\gamma_{Q}\right)
\end{aligned}
$$

and

$$
\begin{gathered}
\gamma_{q}=\frac{q_{0} l^{3}}{2 \sqrt{E I_{z} G I_{t}}} \quad \gamma_{Q}=\frac{Q_{0} l^{2}}{\sqrt{E I_{z} G I_{t}}} \quad K=\frac{\pi}{l} \sqrt{\frac{E I_{w}}{G I_{t}}} \\
\delta_{y}=\frac{\beta_{y}}{l} \sqrt{\frac{E I_{z}}{G I_{t}}} \quad \varepsilon_{q}=\frac{z_{q}-z_{S}}{l} \sqrt{\frac{E I_{z}}{G I_{t}}} \\
\varepsilon_{Q}=\frac{z_{Q}-z_{S}}{l} \sqrt{\frac{E I_{z}}{G I_{t}}}
\end{gathered}
$$

are non-dimensional parameters, very much identical to the ones proposed by Anderson and Trahair [15].

Problem D (Strong Form). Find $\lambda \in \mathrm{R}$ and $\bar{\phi} \in C^{4}([0,1])$, with $\bar{\phi} \neq 0$, satisfying the differential equation

$$
\begin{gathered}
-\mu_{0}^{f 2} \bar{\phi} \lambda^{2}+\left(2 \varepsilon_{q} \gamma_{q} \bar{\phi}-\delta_{y}\left(\mu_{0}^{f} \bar{\phi}_{, \xi}\right), \xi\right) \lambda-\bar{\phi}_{, \xi \xi} \\
+\frac{K^{2}}{\pi^{2}} \bar{\phi}_{, \xi \xi \xi \xi}=0
\end{gathered}
$$


in $(0,1)$ and the boundary conditions

$$
\begin{aligned}
& \bar{\phi}(0)=0 \quad \varepsilon_{Q} \gamma_{Q} \bar{\phi}(1) \lambda+\bar{\phi}_{, \xi}(1)-\frac{K^{2}}{\pi^{2}} \bar{\phi}_{, \xi \xi \xi}(1)=0 \\
& \bar{\phi}_{, \xi}(0)=0(\mathrm{NW}) \quad \text { or } \quad \bar{\phi}_{, \xi \xi}(0)=0(\mathrm{FW}) \quad \bar{\phi}_{, \xi \xi}(1)=0 .
\end{aligned}
$$

Again, it is readily seen that an equivalent variational characterisation can be provided for Problems $\mathrm{C}$ and D. For instance, $(14)$ and $(15),\left(16_{2}\right),\left(17_{2}\right),\left(18_{2}\right),\left(19_{2}\right)$ and $\left(19_{1}\right)$ (the last one only if the fixed end section is free to warp- $-\mathrm{FW})$ are the Euler-Lagrange equations and natural boundary conditions related to the vanishing of the first variation of the functional

$$
\begin{aligned}
\delta^{2} \bar{\Pi}= & \frac{1}{2} \int_{0}^{1} \bar{v}_{, \xi \xi}^{2} \mathrm{~d} \xi+\frac{1}{2} \int_{0}^{1} \bar{\phi}_{, \xi}^{2} \mathrm{~d} \xi+\frac{K^{2}}{2 \pi^{2}} \int_{0}^{1} \bar{\phi}_{, \xi \xi}^{2} \mathrm{~d} \xi \\
& +\left(\frac{1}{2} \int_{0}^{1} \mu_{0}^{f}\left(2 \bar{v}_{, \xi \xi} \bar{\phi}+\delta_{y} \bar{\phi}_{, \xi}^{2}\right) \mathrm{d} \xi+\varepsilon_{q} \gamma_{q} \int_{0}^{1} \bar{\phi}^{2} \mathrm{~d} \xi\right. \\
& \left.+\frac{1}{2} \varepsilon_{Q} \gamma_{Q} \bar{\phi}(1)^{2}\right) \lambda
\end{aligned}
$$

whose admissible arguments $\bar{v}, \bar{\phi}$ (i) lie in the space of squareintegrable functions in $(0,1)$ with equally square-integrable first and second derivatives and (ii) satisfy $\left(16_{1}\right),\left(17_{1}\right),\left(18_{1}\right)$ and, when warping is prevented at the fixed end section ( $\mathrm{NW})$, also $\left(19_{1}\right)$ - note that

$\delta^{2} \bar{\Pi}=\frac{l}{G I_{t}} \delta^{2} \Pi$.

Once $\lambda_{c r}$ has been obtained, the elastic critical moment is given simply by

$M_{c r}=\frac{\sqrt{E I_{z} G I_{t}}}{l}\left(\gamma_{q}+\gamma_{Q}\right) \lambda_{c r}$.

From either of the above two problems, we conclude that "cantilever families" sharing the same (i) $K, \delta_{y}, \varepsilon_{q}, \varepsilon_{Q}$ and $q_{0} l / Q_{0}$ values and (ii) warping restraint condition at the fixed end ( $\mathrm{NW}$ or $\mathrm{FW}$ ) exhibit identical values of $\gamma_{q} \lambda$ or $\gamma_{Q} \lambda$ at buckling. In other words, the cantilevers belonging to such "families" are similar, as far as the LTB behaviour is concerned-thus, $\gamma_{q} \lambda_{c r}$ or $\gamma_{Q} \lambda_{c r}$ can be written as functions of $K, \delta_{y}, \varepsilon_{q}, \varepsilon_{Q}$ and $q_{0} l / Q_{0}$.

It is worth pointing out that the same conclusion can be reached through the use of Buckingham's $\pi$-theorem [34], which summarises the entire theory of dimensional analysisBuckingham himself did not rigorously prove the theorem that bears his name, although he presented evidence making its validity seem plausible (a proof of this theorem can be found, for instance, in [35]). On the basis of a purely physical (i.e. phenomenological) reasoning, the following unspecified functional relationship, involving $n=9$ quantities, may be stated:

$M_{c r}=f\left(E I_{z}, G I_{t}, E I_{w}, \beta_{y}, l,\left(z_{q}-z_{S}\right),\left(z_{Q}-z_{S}\right), \frac{q_{0} l}{Q_{0}}\right)$.

The rank of the associated dimensional matrix is $r=3$ and Buckingham's $\pi$-theorem asserts that (29) can be written in terms of a complete set of $n-r=6$ non-dimensional parameters:

$\pi_{1}=g\left(\pi_{2}, \pi_{3}, \pi_{4}, \pi_{5}, \pi_{6}\right)$.

There is an infinite number of different complete sets of nondimensional parameters. One possible choice is $\pi_{1}=\gamma_{q} \lambda_{c r}$ (or, alternatively, $\pi_{1}=\gamma_{Q} \lambda_{c r}$ ), $\pi_{2}=K, \pi_{3}=\delta_{y}, \pi_{4}=$ $\varepsilon_{q}, \pi_{5}=\varepsilon_{Q}$ and $\pi_{6}=q_{0} l / Q_{0}$, which arises naturally during the manipulation of the governing equations.

It should be stressed that, up to this point, the shape of the cross-section has not yet been invoked in any of the derivations. Thus, all the previous results are valid for thinwalled cantilevers with arbitrary singly symmetric open crosssections under major axis bending.

Since the parameters $K, \delta_{y}, \varepsilon_{q}, \varepsilon_{Q}$ do not convey a clear perception of the cantilever slenderness, asymmetry and load position, we adopt a new set of non-dimensional parameters, which (i) apply specifically to I-section beams and (ii) are closely related to the geometrical and mechanical properties governing their LTB behaviour. They are similar to the ones proposed by Kitipornchai and Trahair [36] and given by

$$
\begin{aligned}
\bar{K} & =\frac{\pi}{l} \sqrt{\frac{E I_{z} h_{S}^{2}}{4 G I_{t}}} \quad \psi_{f}=\frac{I_{b f}-I_{t f}}{I_{b f}+I_{t f}} \quad \zeta_{q}=2 \frac{z_{q}-z_{S}}{h_{S}} \\
\zeta_{Q} & =2 \frac{z_{Q}-z_{S}}{h_{S}},
\end{aligned}
$$

where $h_{S}$ is the distance between the flange mid-lines and $I_{b f}$ and $I_{t f}$ are the moments of inertia, about the $z$-axis, of the bottom and top flanges. It is worth noting that:

(i) The parameter $\psi_{f}\left(i_{1}\right)$ is purely geometrical (i.e. independent of the $M_{y}^{f}$ sign), unlike the ones defined in [5, 37], (i $i_{2}$ ) provides an easy-to-feel measure of the flange asymmetry and $\left(\mathrm{i}_{3}\right)$ varies from $\psi_{f}=-1$ (T-section) to $\psi_{f}=1$ (inverted T-section), with $\psi_{f}=0$ for an equal flanged I-section. It is related to the warping constant $I_{w}$ through [36,37]

$I_{w}=\left(1-\psi_{f}^{2}\right) I_{z} h_{S}^{2} / 4$

and may also be used to approximate $\beta_{y}$ by means of [37] $\beta_{y} \approx$

$\begin{cases}-0.8 \psi_{f} h_{S} & \text { if } \psi_{f}>0 \text { (i.e. favourable flange asymmetry) } \\ -\psi_{f} h_{S} & \text { if } \psi_{f}<0 \text { (i.e. unfavourable flange asymmetry) }\end{cases}$

(note that $\beta_{y}$ obviously vanishes for an equal flanged I-section). An assessment of the errors involved in the approximation (33) and in the subsequent evaluation of $\gamma_{q} \lambda_{c r}$ and $\gamma_{Q} \lambda_{c r}$ is presented in an appendix at the end of this paper.

(ii) In commonly used I-section cantilevers, the beam parameter $\bar{K}$ ranges from 0.1 to $2.5[17,36]$. Low (high) $\bar{K}$ values mean long (short) cantilevers and/or compact (slender) cross-sections. Furthermore, one has $\bar{K}=K$ for an equal flanged cantilever $\left(\psi_{f}=0\right)$.

(iii) The load position parameters $\zeta_{q}$ and $\zeta_{Q}$ enable an easy visualisation of the location of the load point of application 
along the cross-section height (or, to be exact, its location in between the flange mid-lines). Typical values of these parameters are $\left(\mathrm{iii}_{1}\right) \zeta_{q(Q)}=0$ (load applied at the shear centre), (iii 2$) \zeta_{q(Q)}=-\left(1+\psi_{f}\right)$ (load applied at the top flange centroid) and (iii 3$) \zeta_{q(Q)}=1-\psi_{f}$ (load applied at the bottom flange centroid) [17].

(iv) Since the set $\left\{K, \delta_{y}, \varepsilon_{q}, \varepsilon_{Q}\right\}$ can be uniquely determined from $\left\{\bar{K}, \psi_{f}, \zeta_{q}, \zeta_{Q}\right\}$ by means of the expressions

$$
\begin{gathered}
K=\bar{K} \sqrt{1-\psi_{f}^{2}} \quad \varepsilon_{q(Q)}=\frac{1}{\pi} \bar{K} \zeta_{q(Q)} \\
\delta_{y} \approx \begin{cases}-\frac{2 \times 0.8}{\pi} \psi_{f} \bar{K} & \text { if } \psi_{f}>0 \\
-\frac{2}{\pi} \psi_{f} \bar{K} & \text { if } \psi_{f}<0,\end{cases}
\end{gathered}
$$

the latter can also be used to write the governing equations in non-dimensional form.

\section{Numerical implementation}

The application of a structure-preserving discretisation method (such as the methods of Rayleigh-Ritz and BubnovGalerkin) to Problem D generates an algebraic quadratic eigenvalue problem of the form

$$
\left(\boldsymbol{A}+\lambda \boldsymbol{B}+\lambda^{2} \boldsymbol{C}\right) \boldsymbol{x}=\mathbf{0},
$$

where $\boldsymbol{A}, \boldsymbol{B}$ and $\boldsymbol{C}$ are real symmetric matrices (of order $n$, say) $-\boldsymbol{A}$ and $\boldsymbol{C}$ are positive and negative definite, respectively. In the case of small-to-moderate $n$, the standard approach to solve (36) numerically involves its linearisation, i.e. its conversion into an equivalent linear generalised eigenproblem by means of an augmentation procedure resembling the reduction of a second-order linear differential equation to a system of two first-order ones [38,39]. In practice, the most commonly used linearisations involve one of the companion forms

$$
\begin{aligned}
& {\left[\begin{array}{c|c}
\mathbf{0} & \boldsymbol{N} \\
\hline-\boldsymbol{A} & -\boldsymbol{B}
\end{array}\right]\left[\begin{array}{l}
\boldsymbol{x} \\
\hline \boldsymbol{u}
\end{array}\right]=\lambda\left[\begin{array}{c|c}
\boldsymbol{N} & \mathbf{0} \\
\hline \mathbf{0} & \boldsymbol{C}
\end{array}\right]\left[\begin{array}{c}
\boldsymbol{x} \\
\hline \boldsymbol{u}
\end{array}\right]} \\
& {\left[\begin{array}{c|c}
-\boldsymbol{A} & \mathbf{0} \\
\hline \mathbf{0} & \boldsymbol{N}
\end{array}\right]\left[\frac{\boldsymbol{x}}{\mathrm{u}}\right]=\lambda\left[\begin{array}{c|c}
\boldsymbol{B} & C \\
\hline N & \mathbf{0}
\end{array}\right]\left[\frac{x}{u}\right],}
\end{aligned}
$$

where $\boldsymbol{N}$ is any non-singular $n \times n$ real matrix and $\boldsymbol{u}=\lambda \boldsymbol{x}$. This approach has two major drawbacks: (i) the dimension of the linearised problem is twice that of the original quadratic one and (ii) backward stability cannot always be guaranteed for the quadratic problem, even if a backward stable algorithm is used to solve the linearised problem [40].

Therefore, we opted for the discretisation of Problem C, leading directly to an algebraic linear generalised eigenproblem of the form

$\boldsymbol{G x}=\frac{1}{\lambda} \boldsymbol{K} \boldsymbol{x}$,

where $\boldsymbol{G}$ and $\boldsymbol{K}$ are real symmetric matrices and $\boldsymbol{K}$ is positive definite. Then, it is possible to perform the Cholesky factorisation of $\boldsymbol{K}\left(\boldsymbol{K}=\boldsymbol{L} \boldsymbol{L}^{\mathrm{T}}\right.$, where $\boldsymbol{L}$ stands for a lower triangular matrix with positive diagonal entries) and reduce (39) to the standard symmetric form

$\left(\boldsymbol{L}^{-1} \boldsymbol{G} \boldsymbol{L}^{-\mathrm{T}}\right) \boldsymbol{y}=\frac{1}{\lambda} \boldsymbol{y}$,

where $\boldsymbol{y}=\boldsymbol{L}^{\mathrm{T}} \boldsymbol{x}$. The standard problem (40) can be solved by the symmetric $Q R$ algorithm (e.g., [41]) or any other eigensolver for real symmetric matrices.

The discretisation scheme employed in this work is the Rayleigh-Ritz method, named after Strutt, 3rd Baron Rayleigh, and Ritz [42,43] - for a comprehensive analysis of the method and a broad discussion of its application areas, see works by Mikhlin $[32,44]$. The functions $\bar{v}$ and $\bar{\phi}$ were approximated by the sequences of linear combinations of coordinate functions

$$
\begin{gathered}
\bar{v}_{n}(\xi)=\sum_{k=1}^{n} a_{k}^{(n)}\left[1-\cos \left(\frac{(2 k-1) \pi}{2} \xi\right)\right] \\
n=1,2, \ldots \\
\bar{\phi}_{n}(\xi)=\sum_{k=1}^{n} b_{k}^{(n)}\left[1-\cos \left(\frac{(2 k-1) \pi}{2} \xi\right)\right] \\
n=1,2, \ldots(\mathrm{NW}) \\
\bar{\phi}_{n}(\xi)=b_{1}^{(n)} \xi+\sum_{k=2}^{n} b_{k}^{(n)} \sin ((k-1) \pi \xi) \\
n=1,2, \ldots(\mathrm{FW}),
\end{gathered}
$$

where the superscript in the coefficients $a_{k}^{(n)}, b_{k}^{(n)}$ stresses their dependence on $n$. Since the same number $n$ of terms is used to approximate $\bar{v}$ and $\bar{\phi}$, one is led to a discrete problem with $2 n$ degrees of freedom-note also that (43) satisfies the natural boundary condition $\left(19_{1}\right)$. The numerical procedure was continued until the relative differences between the buckling capacities $\gamma_{q} \lambda_{c r}$ or $\gamma_{Q} \lambda_{c r}$ computed with three consecutive values of $n$ did not exceed $0.1 \%$ - it was found that this criterion could always be satisfied by considering up to nine terms $(n \leq 9)$ in (41)-(43).

\section{Parametric study}

A parametric study was conducted to determine the nondimensional elastic buckling capacities $\gamma_{q} \lambda_{c r}$ or $\gamma_{Q} \lambda_{c r}$ of I-section cantilevers and the associated normalised buckling mode shapes $\bar{\phi}_{c r}$ as a function of the beam parameter $\bar{K}(0.1 \leq$ $\bar{K} \leq 2.5$ ), for (i) selected values of the flange asymmetry parameter $\psi_{f}$ (in the range $-0.8 \leq \psi_{f} \leq 0.8$ ) and (ii) three load positions (shear centre, top and bottom flange centroids). The cantilevers were acted on by a uniform load or a tip load (i.e. $Q_{0}=0$ or $q_{0}=0$ ) - the simultaneous application of the two loads was not considered in this work. As discussed earlier, warping at the fixed end section was either fully prevented (NW) or completely free (FW).

The numerical results were compared with values reported by (i) Wang and Kitipornchai [17], for cantilevers with no warping at the fixed end and both equal and unequal flanges, and by (ii) Trahair [12], for cantilevers free to warp at the fixed 

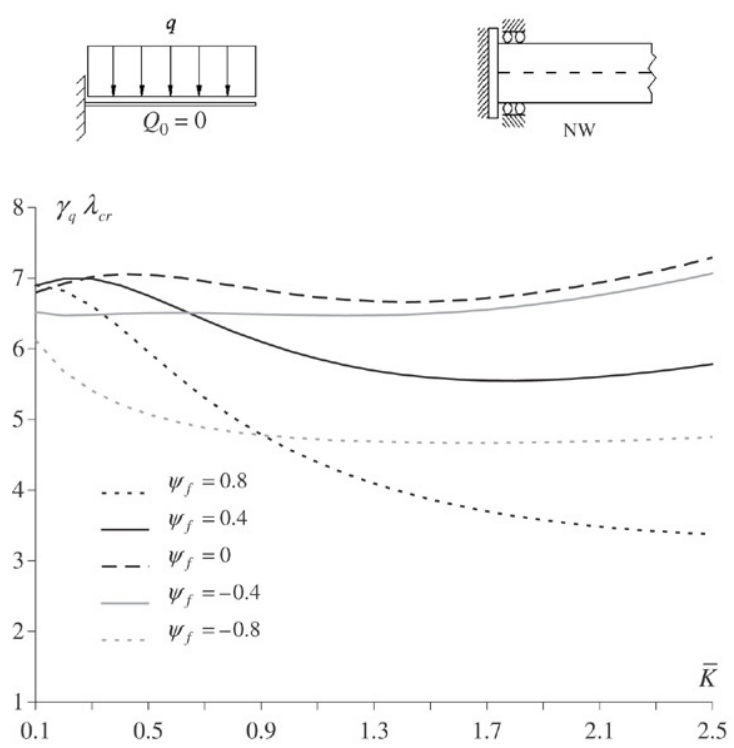
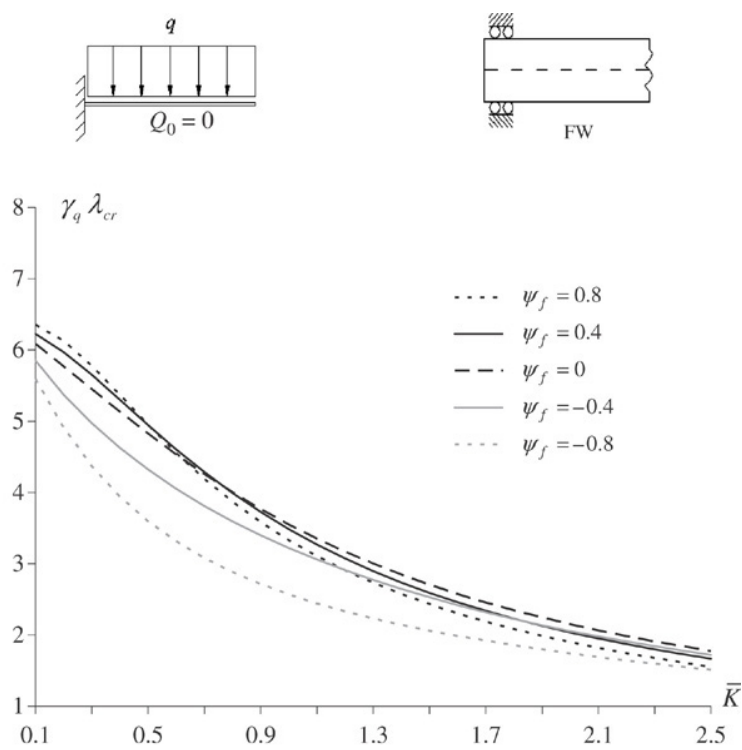

Fig. 2. Cantilevers acted on by a uniform load applied at the top flange centroid $\left(\zeta_{q}=-1-\Psi_{f}\right)$ : variation of $\gamma_{q} \lambda_{c r}$ with $\bar{K}$ and $\Psi_{f}$.
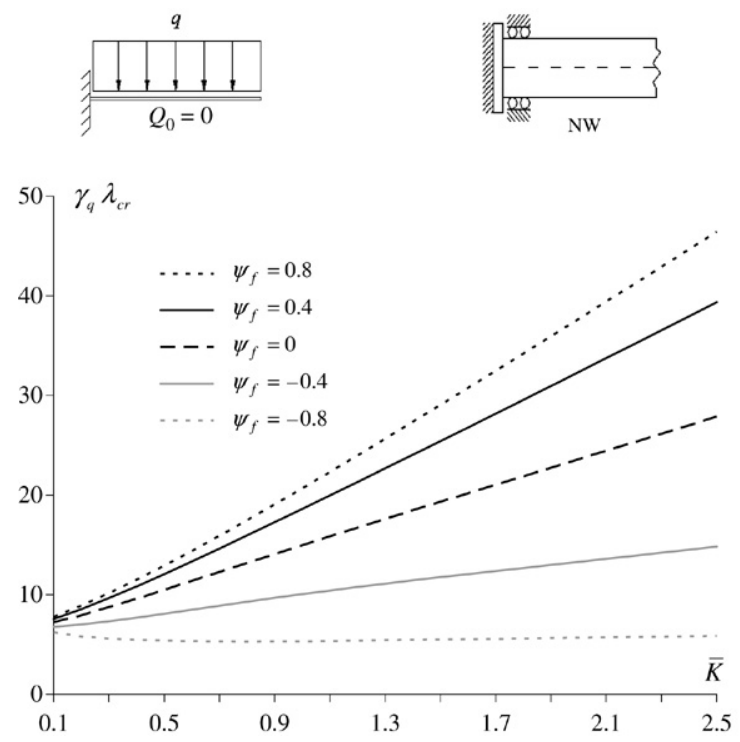
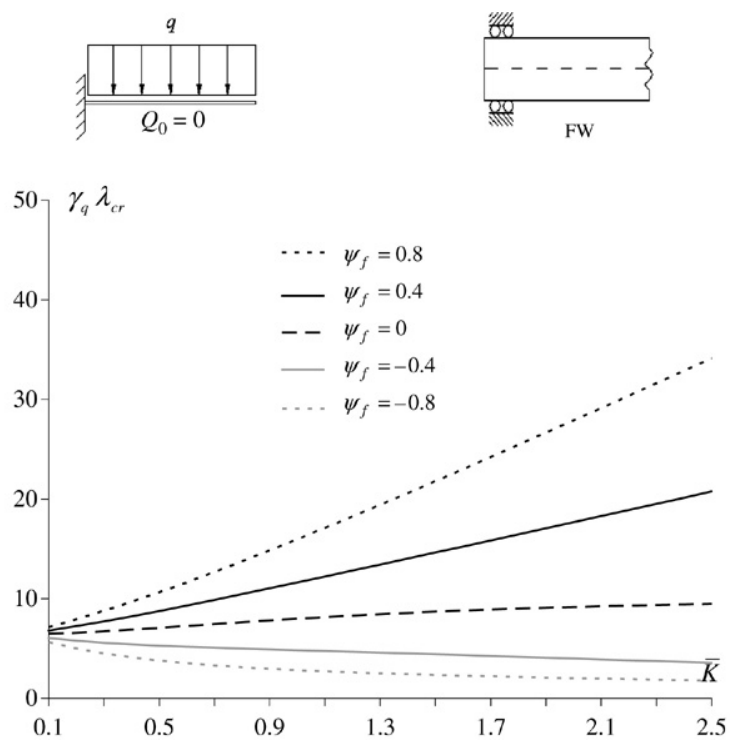

Fig. 3. Cantilevers acted on by a uniform load applied at the shear centre $\left(\zeta_{q}=0\right)$ : variation of $\gamma_{q} \lambda_{c r}$ with $\bar{K}$ and $\Psi_{f}$.

end and equal flanges - to the best of our knowledge, no $M_{c r}$ values for cantilevers with unequal flanges and free to warp at the fixed end are available in the literature. An excellent agreement was found in all cases.

Figs. 2-7 show plots of the elastic buckling capacities $\gamma_{q} \lambda_{c r}$ and $\gamma_{Q} \lambda_{c r}$ against the beam parameter $\bar{K}$, for selected values of the flange asymmetry parameter $\psi_{f}$ and the three load positions considered. These plots show that:

(i) As expected, the cantilevers restrained from warping at the fixed end (NW) exhibit higher buckling capacities than the ones that are free to warp at that section (FW). While the difference is only marginal for very low values of $\bar{K}$, it gradually increases with this parameter and becomes substantial for high $\bar{K}$ values. This indicates that the buckling capacity of a cantilever restrained from warping at the support and having a high $\bar{K}$ depends, to a large extent, on the warping torsion generated during bucklingthis effect is hardly mobilised in the absence of an external warping restraint. On the other hand, the warping torsion is not relevant in cantilevers with very low $\bar{K}$, which implies that the warping restraint at the support has little impact on $\gamma_{q} \lambda_{c r}$ or $\gamma_{Q} \lambda_{c r}$

(ii) Although the plots concerning cantilevers subjected to uniform and tip loads display similar qualitative features, the $\gamma_{q} \lambda_{c r}$ values are always higher: for equal end values (at $\xi=0$ and $\xi=1$ ), the magnitude of the non-dimensional bending moment distribution $\left|\mu_{0}^{f}(\xi) \lambda\right|$ associated with the uniform load is smaller at any given interior point $(0<$ $\xi<1)$.

(iii) The so-called Wagner's effect $[15,45]$, which may be viewed as an increase (reduction) in the actual torsional rigidity of the cross-sections when the compression 

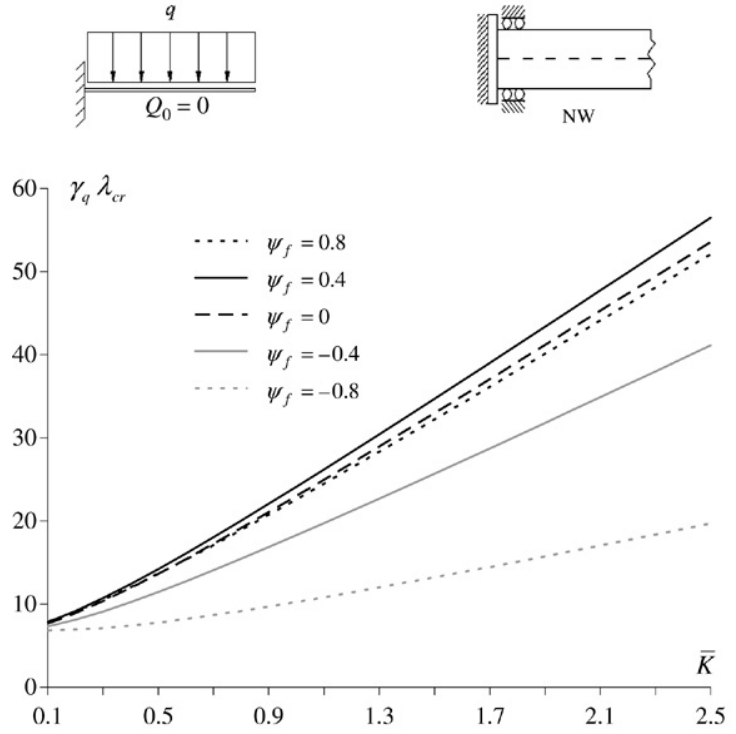
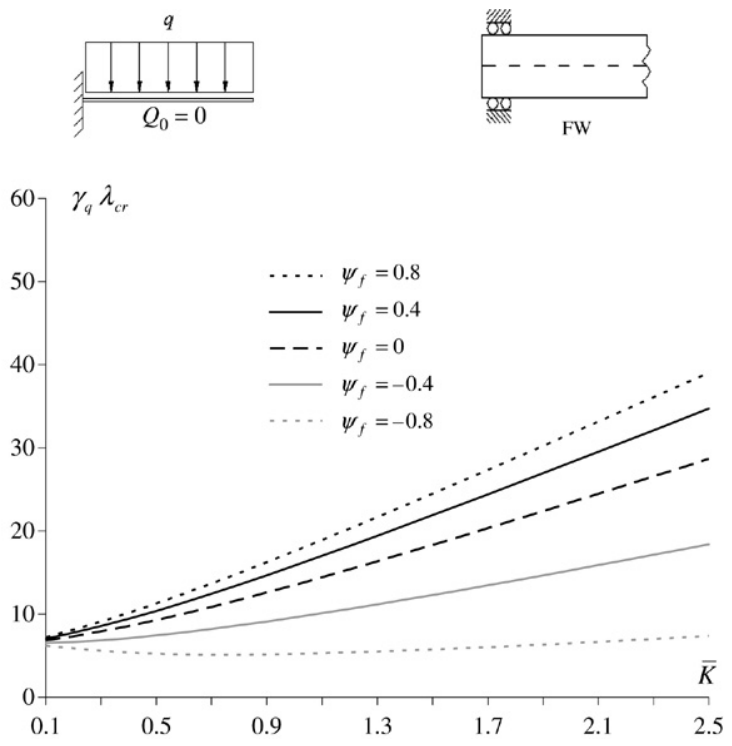

Fig. 4. Cantilevers acted on by a uniform load applied at the bottom flange centroid ( $\zeta_{q}=1-\Psi_{f}$ ): variation of $\gamma_{q} \lambda_{c r}$ with $\bar{K}$ and $\Psi_{f}$.

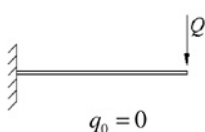

$q_{0}=0$

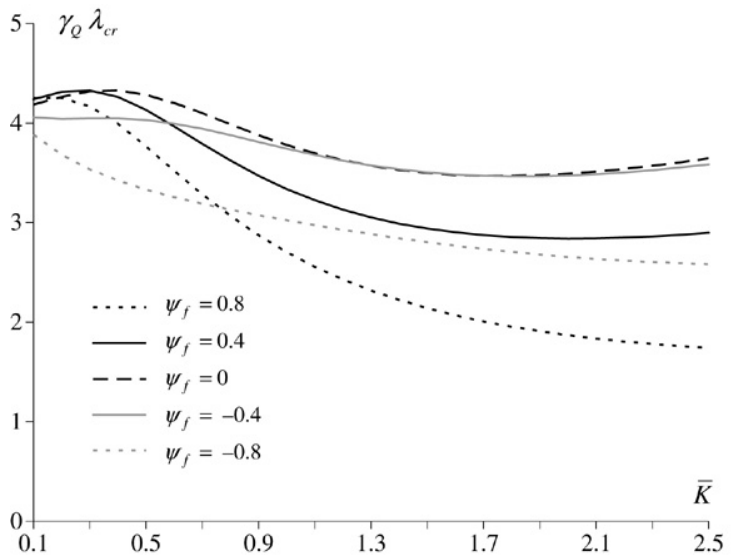

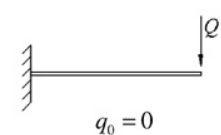
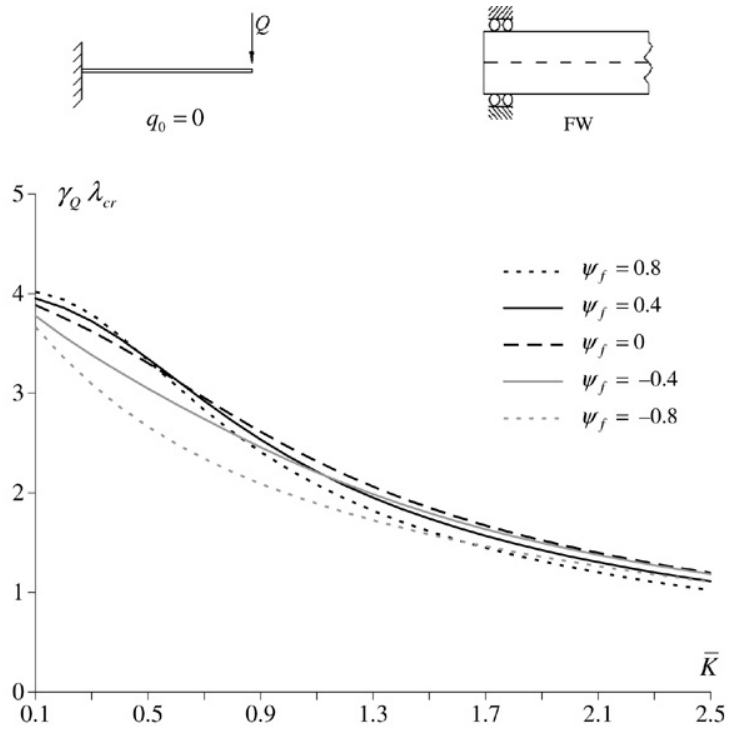

Fig. 5. Cantilevers acted on by a tip load applied at the top flange centroid $\left(\zeta_{Q}=-1-\Psi_{f}\right.$ ): variation of $\gamma_{Q} \lambda_{c r}$ with $\bar{K}$ and $\Psi_{f}$.

flange is larger (smaller) than the tension one, is clearly noticeable for the cantilevers loaded at the shear centre: both $\gamma_{q} \lambda_{c r}$ and $\gamma_{Q} \lambda_{c r}$ increase monotonically with $\psi_{f}$.

(iv) In the cantilevers loaded at the top or bottom flange, the variation of the buckling capacity with $\psi_{f}$ stems from the interplay between (iv 1$)$ Wagner's effect and (iv $\left.{ }_{2}\right)$ the location of the point of load application relative to the shear centre-recall that, for top or bottom flange loading, the value of the parameters $\zeta_{q}$ and $\zeta_{Q}$ depends on $\psi_{f}$. This explains why, in some cases, this variation is not monotonic (e.g., warping restrained cantilevers with $\psi_{f}>0$ and bottom flange loading). It is also worth noting that, for top flange loading and moderate-to-high $\bar{K}$ values, the maximum buckling capacity occurs for sections with equal (or nearly equal) flanges.
The critical buckling mode shapes $\bar{\phi}_{c r}$ of cantilevers with $\bar{K}=1.0$ and acted on by tip loads are shown in Figs. 8-10 (these shapes are normalised so that $\bar{\phi}_{c r \max }=1$ )- since the buckling mode shapes of the cantilevers subjected to uniform loads are qualitatively similar, they are not presented here. The most striking difference between the two graphs in each of these figures concerns the shape of the critical modes near the supported end-while the cantilevers prevented from warping at the support (NW) display a considerable curvature, the ones that are free to warp (FW) exhibit a linear ascending branch, which is an immediate consequence of the natural boundary condition $\bar{\phi}_{, \xi \xi}(0)=0$. It is also interesting to note that, in the cases of shear centre and top flange loading, $\bar{\phi}_{c r}$ increases monotonically with $\xi$, so that the maximum torsional rotation occurs at the cantilever free end. Conversely, for bottom flange 

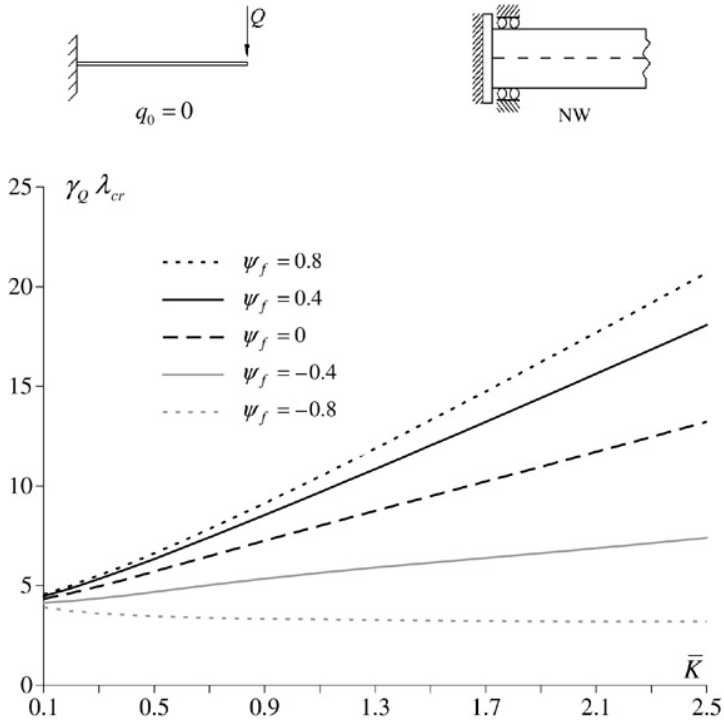
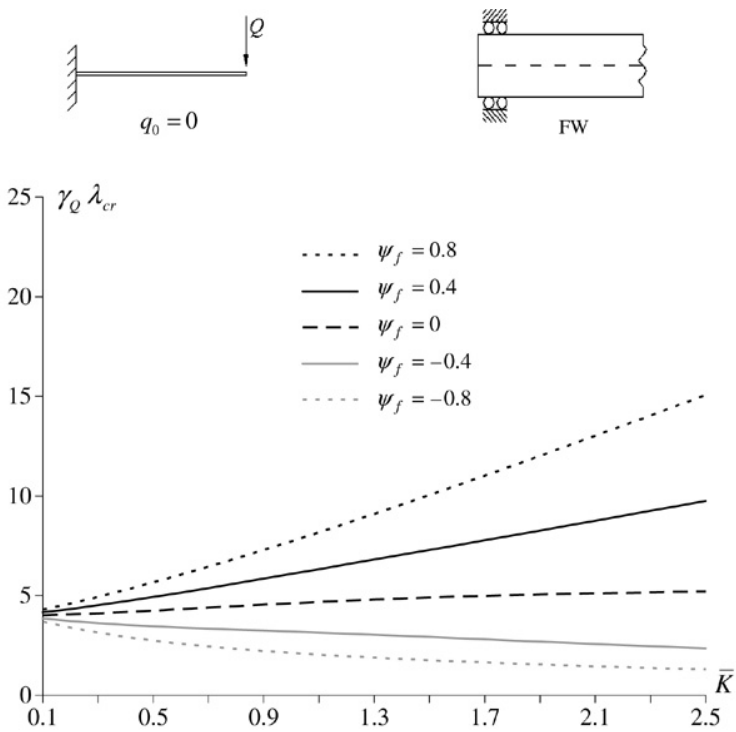

Fig. 6. Cantilevers acted on by a tip load applied at the shear centre $\left(\zeta_{Q}=0\right)$ : variation of $\gamma_{Q} \lambda_{c r}$ with $\bar{K}$ and $\Psi_{f}$.
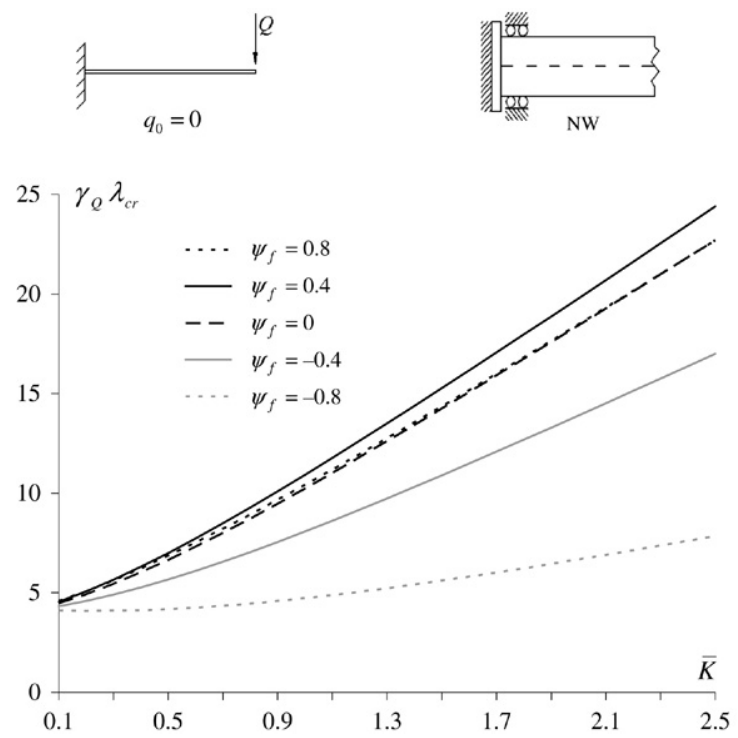
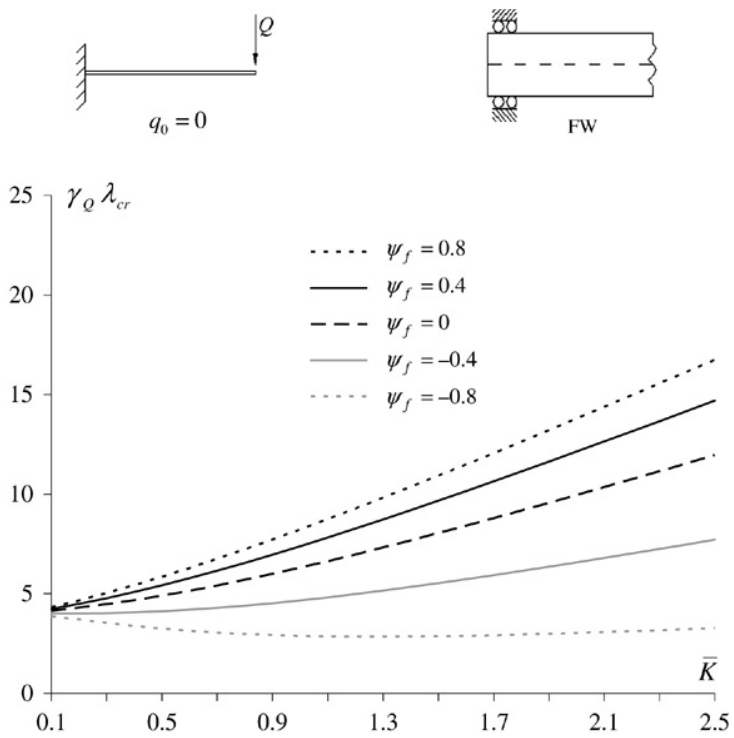

Fig. 7. Cantilevers acted on by a tip load applied at the bottom flange centroid ( $\zeta_{Q}=1-\Psi_{f}$ ): variation of $\gamma_{Q} \lambda_{c r}$ with $\bar{K}$ and $\Psi_{f}$.

loading the critical mode shapes reach a maximum for $\xi<1$ and then decrease towards the cantilever free end, a feature that becomes gradually more significant as the value of the flange asymmetry parameter $\psi_{f}$ diminishes.

\section{Approximate formulae to estimate elastic critical moments}

\subsection{The three-factor formula of Eurocode 3}

One of the most well-known and widely employed formulae to estimate elastic critical moments is the so-called 3-factor formula, which was adopted by the ENV version of Eurocode 3 [5] and retained up until the "Stage 34" Draft of its EN version [37]. It was subsequently set aside, without being replaced (see $[1,46]$ ) — for brief but illuminating accounts of the origin and eventful past of this formula, the interested reader is referred to $[47,48]$. The 3 -factor formula, as it appeared in [5, 37], reads

$$
\begin{aligned}
M_{c r} & =C_{1} \frac{\pi^{2} E I_{z}}{\left(k_{z} L\right)^{2}} \\
& \times\left[\sqrt{\left(\frac{k_{z}}{k_{w}}\right)^{2} \frac{I_{w}}{I_{z}}+\frac{\left(k_{z} L\right)^{2} G I_{t}}{\pi^{2} E I_{z}}+\left(C_{2} z_{g}-C_{3} z_{j}\right)^{2}}-\left(C_{2} z_{g}-C_{3} z_{j}\right)\right] .
\end{aligned}
$$

Because of its long and widespread use (at least in Europe), it was decided to adopt formula (44) and to extend its range of application to cantilevers, by deriving appropriate $C_{i}$ factorsbearing this in mind, it is convenient to make some preliminary observations:

(i) In [5,37], $L$ is defined as the beam length between consecutive laterally restrained cross-sections. When 

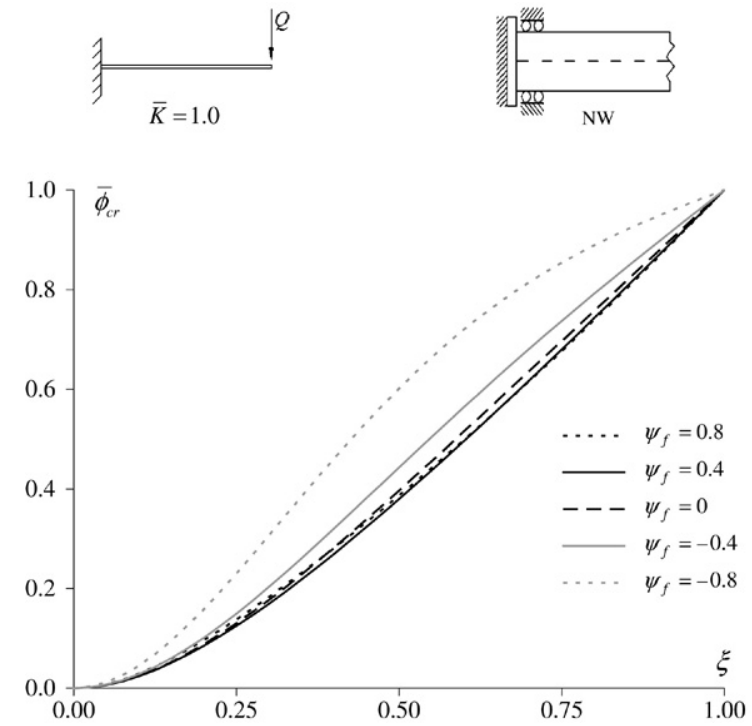
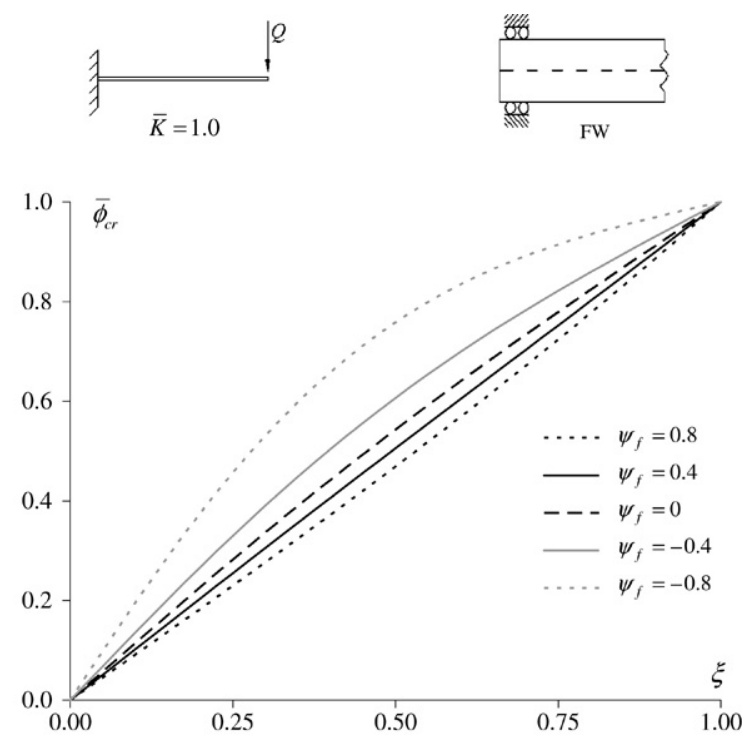

Fig. 8. Cantilevers $(\bar{K}=1.0)$ acted on by a tip load applied at the top flange centroid $\left(\zeta_{Q}=-1-\Psi_{f}\right)$ : normalised critical mode shapes $\bar{\phi}_{c r}$.
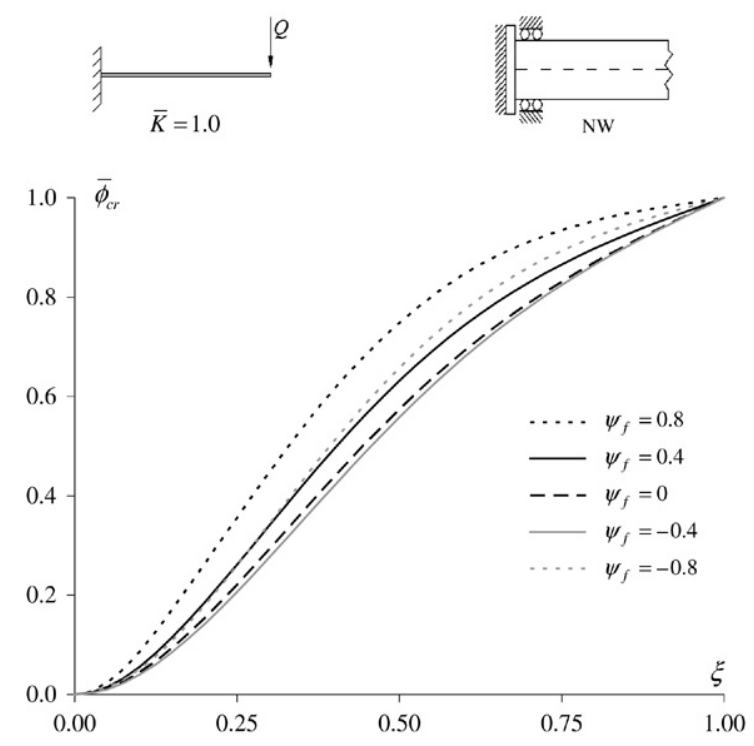
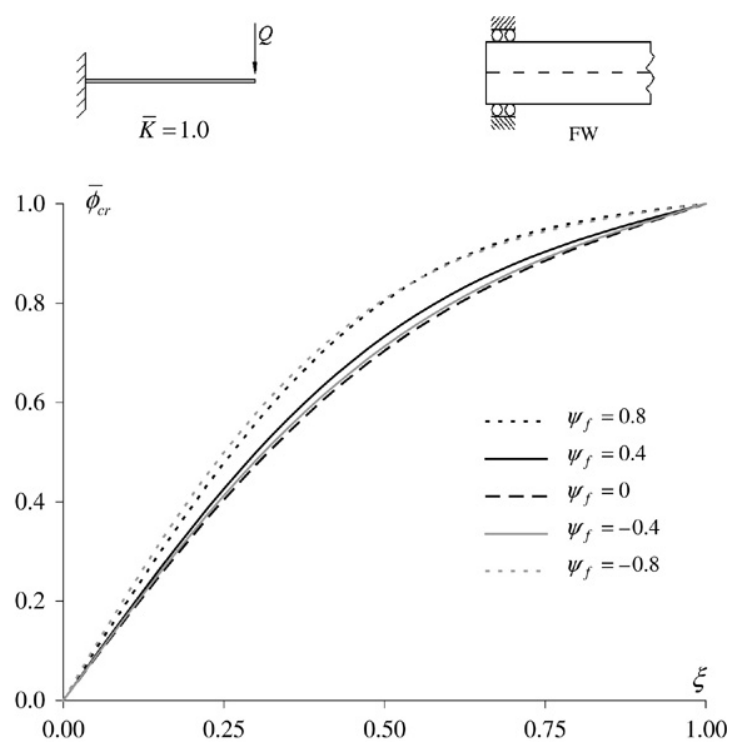

Fig. 9. Cantilevers $(\bar{K}=1.0)$ acted on by a tip load applied at the shear centre $\left(\zeta_{Q}=0\right)$ : normalised critical mode shapes $\bar{\phi}_{c r}$.

dealing with cantilevers, this definition has to be modified and, therefore, we take $L$ as the cantilever length-recall that it was previously denoted in this paper by the lower case letter $l$.

(ii) According to $[5,37]$, the $C_{i}$ factors depend only on (ii $\left.{ }_{1}\right)$ the bending moment diagram and (ii 2 ) the end restraint conditions. However, it has been recognised by several authors (e.g., [13,47,49]) that, except in a few particular cases, these factors also depend on (ii $\left.{ }_{1}\right)$ the beam parameter $\bar{K}$, (ii 2$)$ the location of the point of load application $\left(C_{2}, C_{3}\right)$ and $\left(\mathrm{ii}_{3}\right)$ the degree of cross-section asymmetry with respect to the $y$-axis $\left(C_{3}\right)$.

(iii) The quantities $k_{z}$ and $k_{w}$ are effective length factors, the former associated with the end rotations about the $z$-axis and the latter with the end warping restraint. It should be noticed that these factors (iii 1 ) are not properly defined (there is no direct relation to the distance between inflection points of the critical buckling mode shape $\left\{v_{c r}, \phi_{c r}\right\}$ ) and (iii 2 ) do not fully account for the end support conditions - indeed, when $k_{z}$ changes so do the $C_{i}$ factors. Thus, the values assigned to $k_{z}$ and $k_{w}$ should be regarded as merely conventional.

(iv) For gravity loads, we define $z_{g}=-\left(z_{q(Q)}-z_{S}\right)$, which means that one has $z_{g}>0$ for loads applied above the shear centre and there is no need for any sign conventionincidentally, the convention adopted in [37] is incorrect. Moreover, it should be made clear that formula (44) is not valid when the beam is subjected to transverse loads applied at different levels (e.g., if one has $z_{q} \neq z_{Q}$ ).

(v) The cross-sectional property $z_{j}$ is defined as

$$
z_{j}=z_{S}-\frac{1}{2 I_{y}} \int_{A} z\left(y^{2}+z^{2}\right) \mathrm{d} A=-\frac{\beta_{y}}{2},
$$



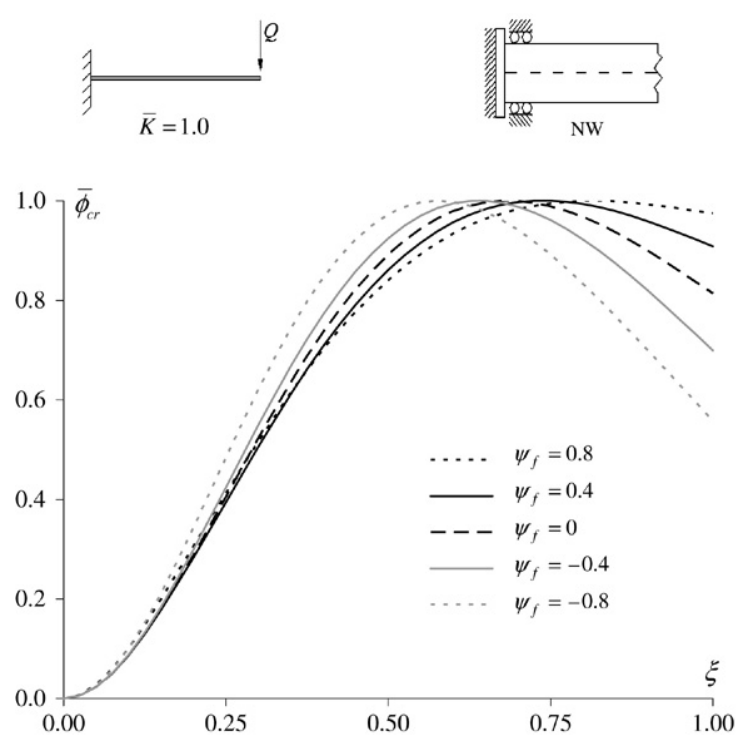
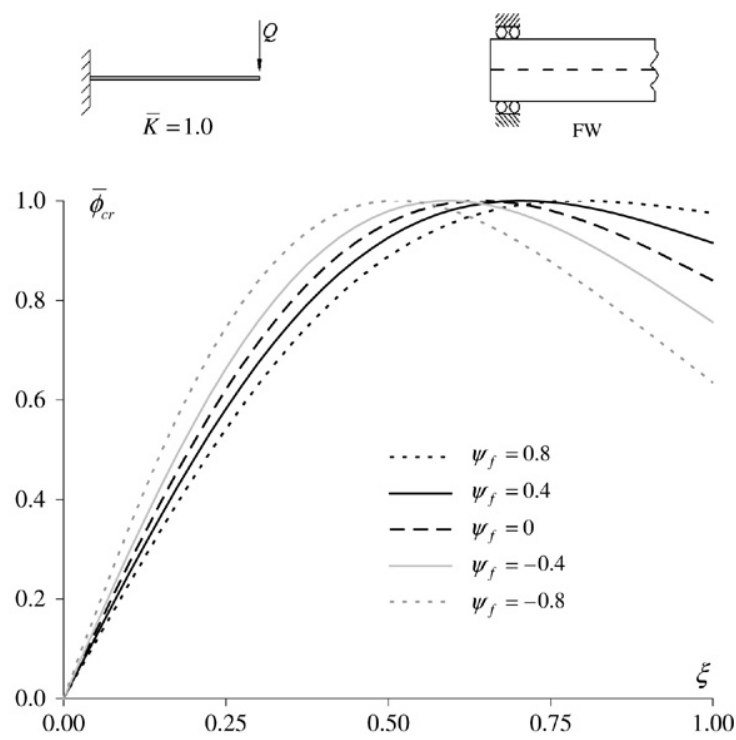

Fig. 10. Cantilevers $(\bar{K}=1.0)$ acted on by a tip load applied at the bottom flange centroid $\left(\zeta_{Q}=1-\Psi_{f}\right)$ : normalised critical mode shapes $\bar{\phi}_{c r}$.

Table 1

Cantilevers with warping prevented at the fixed end (NW) $-C_{1}$ and $C_{2}$ factors $\left(L=l ; k_{z}=2 ; k_{w}=1 ; 0.1 \leq \bar{K} \leq 2.5\right)$

\begin{tabular}{lllr}
\hline & $C_{1}$ & $C_{2}$ & Bottom flange loading \\
\hline Point load & $2.462 / \sqrt{1+\bar{K}^{2}}+2.383 \bar{K} / \sqrt{1+\bar{K}^{2}}$ & $0.380+2.092 \bar{K}-0.318 \bar{K}^{2}$ & $0.512+0.370 \bar{K}_{-0} 0.033 \bar{K}^{2}$ \\
Uniform load & $3.962 / \sqrt{1+\bar{K}^{2}}+5.531 \bar{K} / \sqrt{1+\bar{K}^{2}}$ & $1.130+1.539 \bar{K}-0.176 \bar{K}^{2}$ & $1.049+0.234 \bar{K}_{-0.020 \bar{K}^{2}}$ \\
\hline
\end{tabular}

which means that, once again, no sign convention is needed.

\section{2. $C_{1}, C_{2}$ and $C_{3}$ factors for cantilevers}

The numerical results presented in Section 4 are now used to develop approximate analytical expressions for $C_{1}, C_{2}$ and $C_{3}$, by adopting the following step-by-step strategy:

(i) Fixed values are assigned to the effective lengths factors: $k_{z}=2.0$ (the most intuitive value, in view of the analogy with column flexural buckling) and $k_{w}=1.0$.

(ii) Initially, only the cases corresponding to $z_{g}=z_{j}=0$ (or, equivalently, $\zeta_{q(Q)}=\psi_{f}=0$-i.e. cantilevers with equal flanges and loaded at the shear centre) are dealt with. Then, the non-dimensional version of formula (44) reads

$\gamma_{q(Q)} \lambda_{c r}=\frac{\pi}{2} C_{1} \sqrt{\bar{K}^{2}+1}$.

For the NW case, Figs. 3 and 6 show that the variation of the buckling capacity with $\bar{K}$ is practically linear. Thus, straight lines are fitted to the $\gamma_{q(Q)} \lambda_{c r}$ vs. $\bar{K}$ numerical results - the approximate analytical expressions for $C_{1}$, shown in Table 1, stem from these fits.

For the FW case, on the other hand, a quadratic polynomial is fitted to the $\gamma_{q(Q)} \lambda_{c r} v s . \bar{K}$ numerical results, as shown in Table 3 - it was found that, concerning the statistical parameters qualifying the difference (or distance) between the numerical and proposed values of
$\gamma_{q(Q)} \lambda_{c r}$, this approach yields more accurate estimates than an alternative one that consists of fitting a polynomial of the same degree to tabulated $C_{1} v s . \bar{K}$ data.

(iii) Next, the cases corresponding to $z_{j}=0$ and $z_{g} \neq 0$ (i.e. cantilevers with equal flanges and loaded at the top or bottom flange) are considered. The numerical results given in Section 4 and the $C_{1}$ approximations obtained in the preceding step are used to evaluate $C_{2}$ by means of the expression

$C_{2}=\frac{4 \gamma_{q(Q)}^{2} \lambda_{c r}^{2}-\pi^{2} C_{1}^{2}\left(1+\bar{K}^{2}\right)}{2 \pi C_{1} \gamma_{q(Q)} \lambda_{c r} \bar{K} \zeta_{q(Q)}}$

Polynomials in $\bar{K}$ are then fitted to the tabulated $C_{2}$ vs. $\bar{K}$ values-see Tables 1 and 3 .

(iv) Finally, the cases associated with $z_{j} \neq 0$ (i.e. cantilevers with unequal flanges) lead to the $C_{3}$ values, which are obtained using the approximations for $C_{1}$ and $C_{2}$ developed earlier and the expression

$$
C_{3}=\frac{4 \gamma_{q(Q)}^{2} \lambda_{c r}^{2}-\pi^{2} C_{1}^{2}\left(1+\bar{K}^{2}\left(1-\psi_{f}^{2}\right)\right)-2 \pi C_{1} C_{2} \gamma_{q(Q)} \lambda_{c r} \bar{K} \zeta_{q(Q)}}{2 \pi \times\left\{\begin{array}{l}
0.8 \text { if } \psi_{f}>0 \\
1.0 \text { if } \psi_{f}<0
\end{array}\right\} \times C_{1} \gamma_{q(Q)} \lambda_{c r} \bar{K} \psi_{f}}
$$

Then, curve fitting techniques are again employed to construct polynomials in $\bar{K}$ and $\psi_{f}$ matching the tabulated $C_{3}, \bar{K}$ and $\psi_{f}$ values as closely as possible - see Tables 2 and 4 . It should be noted that the step function appearing 
Table 2

Cantilevers with warping prevented at the fixed end (NW) $-C_{3}$ factor $\left(L=l ; k_{z}=2 ; k_{w}=1 ; 0.1 \leq \bar{K} \leq 2.5 ;-0.8 \leq \psi_{f} \leq 0.8, \psi_{f} \neq 0\right)$

Top flange loading

Point load

Shear centre loading

Bottom flange loading

Top flange loading

Uniform load

Shear centre loading

Bottom flange loading
$1.520-1.342 \psi_{f}-0.010 \psi_{f}^{2}-0.424 \psi_{f}^{3}+\left(0.162+2.419 \psi_{f}+0.875 \psi_{f}^{2}+0.400 \psi_{f}^{3}\right) \bar{K}$ $+\left(0.148-0.623 \psi_{f}-0.216 \psi_{f}^{2}+0.141 \psi_{f}^{3}\right) \bar{K}^{2}$

$1.808-0.944 \psi_{f}+0.299 \psi_{f}^{2}-0.061 \psi_{f}^{3}+\left(0.060+1.235 \psi_{f}-0.574 \psi_{f}^{2}-0.337 \psi_{f}^{3}\right) \bar{K}$ $+\left(0.128-0.409 \psi_{f}+0.047 \psi_{f}^{2}+0.237 \psi_{f}^{3}\right) \bar{K}^{2}$

$1.966-0.792 \psi_{f}+0.139 \psi_{f}^{2}-0.341 \psi_{f}^{3}+\left(0.061+0.549 \psi_{f}+0.077 \psi_{f}^{2}-0.206 \psi_{f}^{3}\right) \bar{K}$ $+\left(0.064-0.135 \psi_{f}-0.050 \psi_{f}^{2}+0.058 \psi_{f}^{3}\right) \bar{K}^{2}$

$2.441-1.589 \psi_{f}+0.176 \psi_{f}^{2}-0.658 \psi_{f}^{3}+\left(-0.412+2.442 \psi_{f}+0.635 \psi_{f}^{2}+\right.$

$\left.0.261 \psi_{f}^{3}\right) \bar{K}+\left(0.273-0.601 \psi_{f}-0.140 \psi_{f}^{2}+0.205 \psi_{f}^{3}\right) \bar{K}^{2}$

$2.609-1.801 \psi_{f}+0.522 \psi_{f}^{2}+0.461 \psi_{f}^{3}+\left(-0.445+2.251 \psi_{f}-0.620 \psi_{f}^{2}-\right.$

$\left.1.443 \psi_{f}^{3}\right) \bar{K}+\left(0.244-0.710 \psi_{f}+0.044 \psi_{f}^{2}+0.611 \psi_{f}^{3}\right) \bar{K}^{2}$

$2.793-1.235 \psi_{f}+0.428 \psi_{f}^{2}-0.630 \psi_{f}^{3}+\left(-0.492+1.008 \psi_{f}-0.134 \psi_{f}^{2}-\right.$

$\left.0.095 \psi_{f}^{3}\right) \bar{K}+\left(0.194-0.263 \psi_{f}-0.003 \psi_{f}^{2}+0.060 \psi_{f}^{3}\right) \bar{K}^{2}$

Table 3

Cantilevers free to warp at the fixed end (FW) $-C_{1}$ and $C_{2}$ factors $\left(L=l ; k_{z}=2 ; k_{w}=1 ; 0.1 \leq \bar{K} \leq 2.5\right)$

\begin{tabular}{llll}
\hline & $C_{1}$ & $C_{2}$ & Bottom flange loading \\
\cline { 3 - 4 } & & Top flange loading & $0.529+0.234 \bar{K}+0.149 \bar{K}^{2}$ \\
Point load & $2.437 / \sqrt{1+\bar{K}^{2}}+0.613 \bar{K} / \sqrt{1+\bar{K}^{2}}-0.105 \bar{K}^{2} / \sqrt{1+\bar{K}^{2}}$ & $0.409+1.444 \bar{K}+0.070 \bar{K}^{2}$ & $1.028+0.388 \bar{K}^{2}+0.150 \bar{K}^{2}$ \\
\hline
\end{tabular}

Table 4

Cantilevers free to warp at the fixed end (FW) $-C_{3}$ factor $\left(L=l ; k_{z}=2 ; k_{w}=1 ; 0.1 \leq \bar{K} \leq 2.5 ;-0.8 \leq \psi_{f} \leq 0.8, \psi_{f} \neq 0\right)$

Top flange loading

Point load

Shear centre loading

Bottom flange loading

Top flange loading

Uniform load

Shear centre loading

Bottom flange loading
$1.732-0.648 \psi_{f}-0.062 \psi_{f}^{2}+0.059 \psi_{f}^{3}+\left(0.066+1.539 \psi_{f}+0.520 \psi_{f}^{2}-0.032 \psi_{f}^{3}\right) \bar{K}$

$+\left(0.446+0.221 \psi_{f}-0.037 \psi_{f}^{2}+0.066 \psi_{f}^{3}\right) \bar{K}^{2}$

$2.021+0.361 \psi_{f}+0.176 \psi_{f}^{2}-0.655 \psi_{f}^{3}+\left(0.242+0.120 \psi_{f}-0.426 \psi_{f}^{2}+0.891 \psi_{f}^{3}\right) \bar{K}$

$+\left(0.337+0.052 \psi_{f}-0.198 \psi_{f}^{2}-0.099 \psi_{f}^{3}\right) \bar{K}^{2}$

$2.156-0.055 \psi_{f}+0.101 \psi_{f}^{2}-0.079 \psi_{f}^{3}+\left(0.435+0.168 \psi_{f}-0.083 \psi_{f}^{2}-0.077 \psi_{f}^{3}\right) \bar{K}$

$+\left(0.238-0.022 \psi_{f}-0.011 \psi_{f}^{2}-0.030 \psi_{f}^{3}\right) \bar{K}^{2}$

$2.669-0.815 \psi_{f}+0.071 \psi_{f}^{2}-0.066 \psi_{f}^{3}+\left(0.113+1.812 \psi_{f}+0.359 \psi_{f}^{2}+0.007 \psi_{f}^{3}\right) \bar{K}$

$+\left(0.499+0.289 \psi_{f}+0.043 \psi_{f}^{2}+0.081 \psi_{f}^{3}\right) \bar{K}^{2}$

$3.036+0.310 \psi_{f}+0.306 \psi_{f}^{2}-0.888 \psi_{f}^{3}+\left(0.066+0.036 \psi_{f}-0.585 \psi_{f}^{2}+1.180 \psi_{f}^{3}\right) \bar{K}$

$+\left(0.462+0.098 \psi_{f}-0.227 \psi_{f}^{2}-0.123 \psi_{f}^{3}\right) \bar{K}^{2}$

$3.277-0.350 \psi_{f}+0.348 \psi_{f}^{2}-0.263 \psi_{f}^{3}+\left(0.190+0.348 \psi_{f}-0.195 \psi_{f}^{2}-0.137 \psi_{f}^{3}\right) \bar{K}$

$+\left(0.395-0.071 \psi_{f}-0.009 \psi_{f}^{2}+0.009 \psi_{f}^{3}\right) \bar{K}^{2}$ in the denominator of (48) is obviously incompatible with the smooth curves provided by continuous polynomials over the whole domain (i.e. for $-0.8 \leq \psi_{f} \leq 0.8$ ). This problem is attenuated by the fact that one has $C_{3} \approx 0$ in the vicinity of the discontinuity (i.e. for $\psi_{f} \approx 0$ ) and its effect on the accuracy of the proposed expressions is only marginal.

All the above curve fittings are performed using standard linear least squares procedures-for a comprehensive and up- to-date treatment of this topic, see [50]. Table 5 provides a synopsis of the statistical parameters measuring the quality (accuracy) of the estimates yielded by the proposed expressions. The degree of the polynomials adopted in the curve fitting process, which, for consistency, was kept the same for similar cases, was selected in order to ensure a targeted accuracy. In view of the plethora of possible accuracy measures, we opted to aim at guaranteeing that the maximum absolute value of the relative error never exceeds $5 \%$, with the relative 
Table 5

Statistical parameters

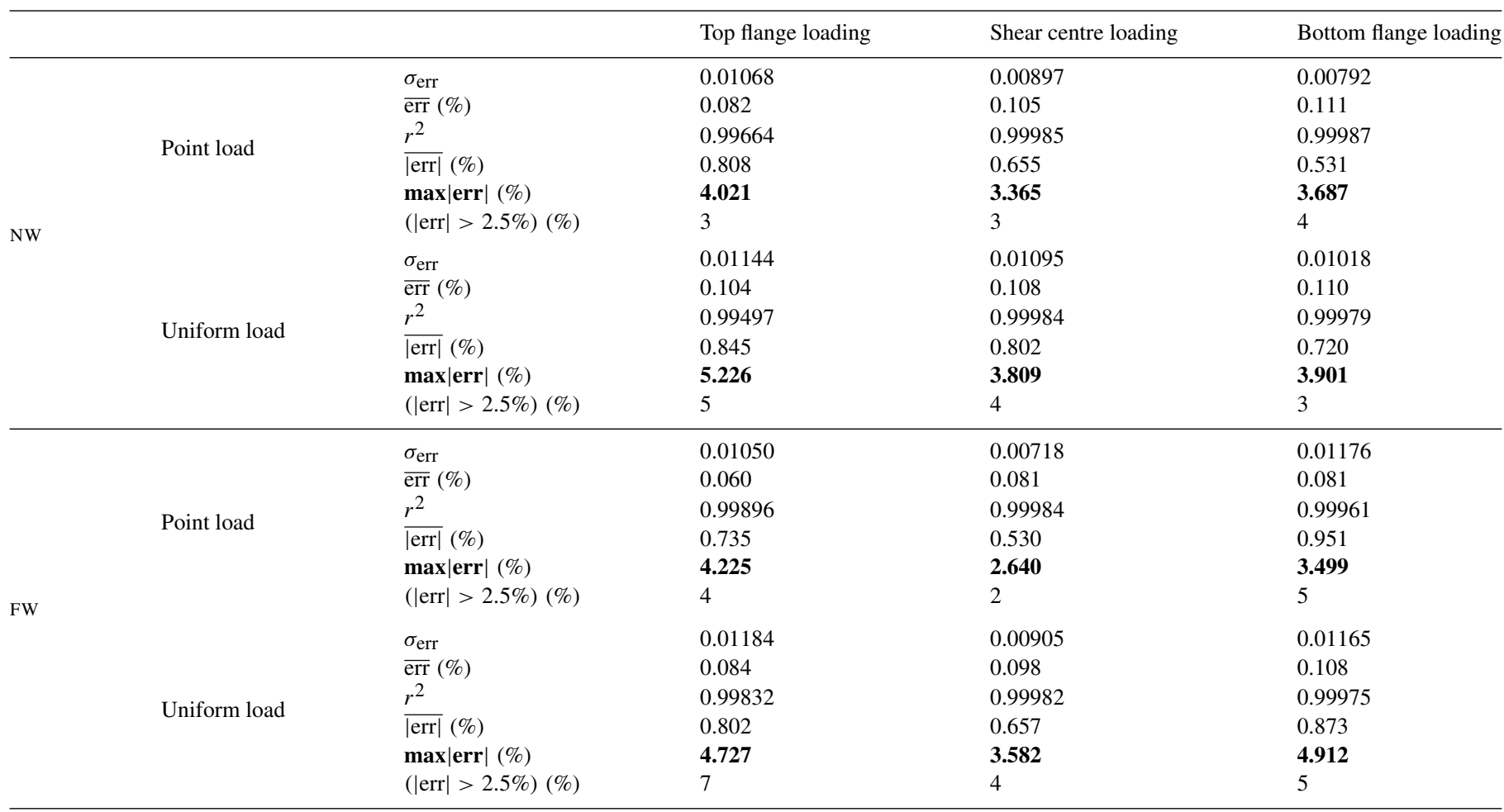

error defined as

err $=\frac{\left(\gamma_{q(Q)} \lambda_{c r}\right)_{\mathrm{num}}-\left(\gamma_{q(Q)} \lambda_{c r}\right)_{\mathrm{app}}}{\left(\gamma_{q(Q)} \lambda_{c r}\right)_{\mathrm{num}}}$

(as shown in Table 5, this criterion is only violated once and by a very minute margin). Table 5 includes (i) the standard deviation $\sigma_{\text {err }}$ and mean value $\overline{\text { err }}$ of the relative error distribution, (ii) the square of the correlation coefficient $r$ (also known as product-moment correlation coefficient or Pearson's correlation coefficient), (iii) the more significant, from a practical viewpoint, mean of the absolute values of the relative error $\overline{|\mathrm{err}|}$, (iv) the maximum absolute value of the relative error and (v) the number of cases, in percentage, with absolute values of the relative error greater than $2.5 \%$-this number is always rather small, with the majority of the cases occurring for $\bar{K} \approx 0.1$ ).

\section{Conclusions}

The so-called 3-factor formula (included, for instance, in the ENV version of Eurocode 3 [5]) is one of the most commonly used general formulae to estimate elastic critical moments in steel beams prone to LTB. In this paper, the domain of application of this formula is extended to cantilevers, by providing approximate analytical expressions to determine the $C_{1}, C_{2}$ and $C_{3}$ factors. The paper deals specifically with I-section cantilevers (i) having equal or unequal flanges, (ii) fully built-in or free to warp at the support and (iii) acted on by uniformly distributed or concentrated tip loads (applied either at the shear centre or at one of the flanges) and includes a careful examination of the influence of the warping restraint condition on the elastic LTB of singly symmetric cantilevers. The estimates yielded by the proposed expressions are very accurate: the differences with respect to the numerical ("exact") results only occasionally exceed $5 \%$.

In summary, our approach consists of the following steps:

(i) write the governing equations in non-dimensional form over a fixed reference domain and, in the process, identify an adequate complete set of non-dimensional parameters;

(ii) implement numerically (i.e. discretise) the continuum model stemming from the preceding step;

(iii) perform a parametric study, on the basis of the nondimensional parameters defined in step (i);

(iv) obtain approximate buckling formulae by fitting a mathematical model to the results of the parametric study using least squares techniques and qualify quantitatively the accuracy of the proposed expressions.

The first step of this procedure is of fundamental importance, since it enables a general and systematic representation of the cantilever LTB behaviour, a deeper insight into the nature of the phenomenon and a reduction in the number of parameters appearing in the governing equations-this last feature bears important consequences, as far as the time and effort that must be put into the parametric study are concerned.

\section{Appendix}

The approximation of $\beta_{y}$ (in terms of $\psi_{f}$ and $h_{S}$ ) given by Eq. (33) is inherent to the results of the parametric study concerning singly symmetric I-section cantilevers (Section 4) 
and, thus, also to the analytical expressions proposed for the $C_{3}$ factor (Section 5.2).

To assess the accuracy of Eq. (33), a number of HEB and IPE sections, having $h_{S}$ values varying between $166 \mathrm{~mm}$ and 484 $\mathrm{mm}$, are made singly symmetric through the modification of one of the flanges, either by reducing its width or by increasing its thickness. The fillets are neglected (i.e. the cross-sections are idealised as a combination of three thin rectangles). The asymmetry parameter $\psi_{f}$ of the modified cross-sections falls within the range $0.10 \leq\left|\psi_{f}\right| \leq 0.95$ - small-to-moderate $\left|\psi_{f}\right|$ values are associated with an increased flange thickness, whereas moderate-to-large $\left|\psi_{f}\right|$ values correspond to a reduced flange width. The approximate $\beta_{y}$ values yielded by Eq. (33) were then compared with the exact ones, as defined by Eq. (2) this comparison shows that:

1. Eq. (33) always overestimates $\beta_{y}$ (if $\psi_{f}>0$, then $\beta_{y \text {,exact }}<$ $\beta_{y \text {, app }}<0$, while $0<\beta_{y}$, exact $<\beta_{y \text {,app }}$ if $\psi_{f}<0$ ). This means that an approximate $\beta_{y}$ value invariably leads to an underestimation of $M_{c r}$.

2. The relative errors $\left(\beta_{y \text {,app }}-\beta_{y \text {,exact }}\right) /\left|\beta_{y \text {, exact }}\right|$ are generally below $15 \%$, even if they occasionally reach almost $20 \%$. However, no obvious correlation appears to exist between the value of $\left|\psi_{f}\right|$ and the magnitude of these relative errors.

At this point, what remains to be assessed is how an error in $\beta_{y}$ affects the evaluation of $M_{c r}$. In order to obtain such an assessment, we considered the worst-case scenario: a $20 \%$ overestimation of $\beta_{y}$, regardless of $\left|\psi_{f}\right|$. It was found that:

(i) As expected, the impact of the $\beta_{y}$ error on $M_{c r}$ grows with $\left|\psi_{f}\right|$ and is more pronounced in the $\mathrm{FW}$ case, due to an increased importance of the Wagner effect in the overall buckling phenomenon.

(ii) Neither the loading nature (tip point load or uniformly distributed load) nor its location (shear centre, top or bottom flange) appear to influence the magnitude of the $M_{c r}$ errors significantly, at least in a consistent way.

(iii) As the beam parameter $\bar{K}$ increases, so does the impact of the $\beta_{y}$ error on $M_{c r}$. In FW cantilevers with highly asymmetric cross-sections $-\left|\psi_{f}\right|=0.8-$, the $\beta_{y}$ error is fully reflected on the evaluation of $M_{c r}$ for $\bar{K}=2.5$. As $\bar{K}$ decreases, this impact is slowly (but steadily) attenuatedit does not exceed $10 \%$ for $\bar{K} \leq 0.5$.

\section{References}

[1] Comité Européen de Normalisation (CEN). Eurocode 3: Design of steel structures, Part 1-1: General rules and rules for buildings (EN 1993-1-1). Brussels; 2005.

[2] British Standards Institution (BSI). BS 5950-1: Structural use of steelwork in buildings. Code of practice for design. Rolled and welded sections. London; 2000.

[3] American Institute of Steel Construction (AISC). Manual of steel construction-Load and resistance factor design. 3rd ed. Chicago; 2001.

[4] Standards Australia (SA). AS 4100: Steel structures (including amendments 1, 2 and 3). Homebush; 1990.

[5] Comité Européen de Normalisation (CEN). Eurocode 3: Design of steel structures, Part 1-1: General rules and rules for buildings (ENV 1993-1-1). Brussels; 1992.

[6] Clark JW, Hill HN. Lateral buckling of beams. J Struct Div 1960;86(7): $175-96$.
[7] Galéa Y. Abaques de déversement pour profilés laminés. Construction Métallique 1981;4:39-51.

[8] Baláz I, Koleková Y. Factors $C_{1}, C_{2}, C_{3}$ for computing the elastic critical moment $M_{c r}$. In: Zborník VI. sympózia Drevo v stavebných konstrukciách so zahranicnou úcast'ou, Kocovce. 2004. p. 29-34.

[9] Timoshenko SP. Einige stabilitätsprobleme der elastizitätstheorie. Z Math Phys 1910;58:337-85. Reprinted in The collected papers of Stephen P. Timoshenko. London: McGraw-Hill; 1953.

[10] Poley S. Lateral buckling of cantilevered I-beams under uniform load. Trans ASCE 1956;121:786-90.

[11] Nethercot DA. The effective lengths of cantilevers as governed by lateral buckling. Struct Eng 1973;51(5):161-8.

[12] Trahair N. Lateral buckling of overhanging beams. In: Morris LJ, editor. Instability and plastic collapse of steel structures. London: Granada; 1983. p. 503-18.

[13] European Commission for Steel and Coal (ECSC). Lateral-torsional buckling in steel and composite beams. Research project 7210-PR-183 final technical report (Book 2-Design guide). 2003.

[14] Doswell B. Lateral-torsional buckling of wide flange cantilever beams. In: Proc. 2002 annual stability conference. Seattle: Structural Stability Research Council; 2002. p. 267-90.

[15] Anderson J, Trahair N. Stability of monosymmetric beams and cantilevers. J Struct Div 1972;98(ST1):269-86.

[16] Roberts TM, Burt CA. Instability of monosymmetric I-beams and cantilevers. Int J Mech Sci 1985;27(5):313-24.

[17] Wang CM, Kitipornchai S. On stability of monosymmetric cantilevers. Eng. Struct. 1986;8(3):169-80.

[18] Wang CM, Kitipornchai S, Thevendran V. Buckling of braced monosymmetric cantilevers. Int J Mech Sci 1987;29(5):321-37.

[19] Attard MM. General non-dimensional equation for lateral buckling. ThinWall Struct 1990;9(1-4):417-35.

[20] Camotim D, Andrade A. Lateral-torsional buckling of I-section cantilevers: Evaluation of critical moments via the EC3 formula. In: Report TC8-2002-023, European convention for constructional steelwork, technical committee 8 (stability) meeting. 2002.

[21] Camotim D, Andrade A. Evaluating critical moments of I-section cantilevers via the EC3 formula: Theoretical background and additional results. In: Report TC8-2003-007, European convention for constructional steelwork, technical committee 8 (stability) meeting. 2003.

[22] Andrade A, Camotim D, Providência P. Lateral-torsional buckling of steel cantilevers: Evaluation of critical moments using the EC3 Formula. In: Proc. 20th czech and slovak national conference on steel structures and bridges. 2003. p. 635-40.

[23] Ciarlet PG. Mathematical elasticity, vol. 1: Three-dimensional elasticity. Amsterdam: Elsevier; 1988.

[24] Vacharajittiphan P, Woolcock ST, Trahair NS. Effect of in-plane deformation on lateral buckling. J Struct Mech 1974;3(1):29-60.

[25] Vlassov B. Thin-walled elastic bars. Jerusalem: Israel Program for Scientific Translations; 1961.

[26] Trahair NS. Flexural-torsional buckling of structures. London: E\&FN Spon (Chapman \& Hall); 1993.

[27] Trefftz E. Über die ableitung der stabilitätskriterien des elastischen gleichgewichts aus der elastizitätstheorie endlicher deformationen. In: Proc. third intern. congr. appl. mech. vol. 3. 1930. p. 44-50.

[28] Trefftz E. Zur theorie der stabilität des elastischen gleichgewichts. Z Angew Math Mech 1933;13(2):160-5.

[29] Knops RJ, Wilkes EW. Theory of elastic stability. In: Truesdell C, editor. Mechanics of solids, vol. 3. Berlin: Springer-Verlag; 1984. p. 125-302. Reissue of Encyclopaedia of physics, vol. VIa/3. Berlin: Springer-Verlag; 1973.

[30] Mikhlin SG. Mathematical physics, an advanced course. Amsterdam: North-Holland; 1970.

[31] Coddington EA, Levinson N. Theory of ordinary differential equations. New York: McGraw-Hill; 1955.

[32] Mikhlin SG. Variational methods in mathematical physics. Oxford: Pergamon Press; 1964.

[33] Lanczos C. The variational principles of mechanics. Toronto: University of Toronto Press; 1970. 
[34] Buckingham E. On physically similar systems; illustrations of the use of dimensional equations. Phys Rev 1914;IV(4):345-76.

[35] Langhaar HL. Dimensional analysis and theory of models. New York: John Wiley \& Sons; 1951.

[36] Kitipornchai S, Trahair NS. Buckling properties of monosymmetric I-beams. J Struct Div 1980;106(ST5):941-57.

[37] Comité Européen de Normalisation (CEN). Eurocode 3: Design of steel structures, Part 1-1: General rules and rules for buildings (prEN 1993-11). Stage 34 draft. Brussels; 2002.

[38] Afolabi D. Linearization of the quadratic eigenvalue problem. Comput Struct 1987;26(6):1039-40.

[39] Tisseur F, Meerbergen K. The quadratic eigenvalue problem. SIAM Rev 2001;43(2):235-86.

[40] Tisseur F. Backward error and condition of polynomial eigenvalue problems. Linear Algebra Appl 2000;309(1-3):339-61.

[41] Golub GH, Van Loan CF. Matrix computations. Baltimore: Johns Hopkins University Press; 1996.

[42] Strutt JW (3rd Baron Rayleigh). The theory of sound, vol. 2. New York: Dover; 1945. Unabridged republication of the second revised and enlarged edition of 1894.

[43] Ritz W. Über eine neue methode zur lösung gewisser variationsprobleme der mathematischen physik. J Reine Angew Math 1908;135:1-61.
Reprinted in Gesammelte werke-oeuvres. Paris: Gauthier-Villars, Société Suisse de Physique; 1911.

[44] Mikhlin SG. The numerical performance of variational methods. Groningen: Wolters-Noordhoff Publishing; 1971.

[45] Wagner H. Torsion and buckling of open sections. NACA technical memorandum 807. 1936 [Translation, by Reiss S, of Verdrehung und knickung von offenen profilen, from the 25th Anniversary Number of the Technische Hochschule; Danzig, 1929. p. 329-43].

[46] Comité Européen de Normalisation (CEN). Eurocode 3: Design of steel structures, Part 1-1: General rules and rules for buildings (prEN 1993-11). Stage 49 draft, Brussels. 2003.

[47] Baláz I, Koleková Y. Critical moments of beams and girdersClark-Mrázik formula. In: Proceedings of the 19th Czech and Slovak national conference on steel structures and bridges. 2000. p. 87-94.

[48] Braham M. Le déversement élastique des poutres en I à section monosymétrique soumises à un gradient de moment de flexion. Constr Métall 2001;(1):17-28.

[49] Baláz I, Koleková Y. Buckling of monosymmetric beams-conjured problem (2 parts). In: Proceedings of the 2nd European conference on steel structures. 1999. p. 701-4. Full paper in CD-ROM.

[50] Björck A. Numerical methods for least squares problems. Philadelphia: SIAM; 1996 